\title{
Micro reactors: principles and applications in organic synthesis
}

\author{
Paul D. I. Fletcher, ${ }^{a}$ Stephen J. Haswell, ${ }^{\text {a,* }}$ Esteban Pombo-Villar, ${ }^{\mathrm{b}}$ Brian H. Warrington, ${ }^{\mathrm{c}}$ \\ Paul Watts, ${ }^{\mathrm{a}}$ Stephanie Y. F. Wong ${ }^{\mathrm{c}}$ and Xunli Zhang ${ }^{\mathrm{a}}$ \\ ${ }^{a}$ Department of Chemistry, Faculty of Science and the Environment, University of Hull, Cottingham Road, Hull HU6 7RX, UK \\ ${ }^{\mathrm{b}}$ Nervous System Research, WSJ-386.07.15, Novartis Pharma Ltd, CH4002 Basel, Switzerland \\ ${ }^{c}$ GlaxoSmithKline Pharmaceuticals, New Frontiers Science Park (North), Third Avenue, Harlow, Essex CM19 5AW, UK
}

Received 12 April 2002

\begin{tabular}{ll}
\multicolumn{1}{c}{ Contents } & \\
1. Introduction & 4735 \\
2. Fabrication and physical characterisation of micro reactors & 4736 \\
2.1. Fabrication & 4736 \\
2.2. Determination of the 3D dimensions of the channel networks & 4737 \\
3. Operation of micro reactors using electrokinetic control & 4739 \\
3.1. Electroosmotic flow (EOF) and electrophoresis & 4739 \\
3.2. Control of reagent mobility in a channel network & 4740 \\
3.3. Reagent mixing and chemical reaction in a micro reactor & 4743 \\
4. Reactions performed in micro reactors & 4745 \\
4.1. Liquid phase reactions & 4745 \\
4.2. Catalytic reactions & 4749 \\
4.3. Gas phase reactions & 4750 \\
5. Potential applications of micro reactors & 4752 \\
6. Concluding remarks & 4754
\end{tabular}

\section{Introduction}

The miniaturisation of chemical reactors offers many fundamental and practical advantages of relevance to today's chemical industry, who are constantly searching for controllable, information rich, high throughput, environmentally friendly methods of producing products with a high degree of chemical selectivity (see, for example, Refs. 1-9 for introductory overviews). Indeed, for some industries such as pharmaceuticals, an informatics based approach, that micro reactor chemistry can uniquely deliver, may be the trigger for a step change in process. In their simplest form, micro reactor devices consist of a network of micron-sized (typical dimensions are in the range 10-300 $\mu \mathrm{m}$ ) channels etched into a solid substrate. For solution-based chemistry, the channel networks are connected to a series of reservoirs containing chemical reagents, products and/or waste to form the complete device

\footnotetext{
Keywords: micro reactors; organic synthesis; fabrication; electrokinetic; reaction modelling.

* Corresponding author. Tel.: +44-1482-465469; fax: +44-1482-466416; e-mail: s.j.haswell@hull.ac.uk
}

or 'chip' (derived from the concept of 'Lab-on-a-Chip') with overall dimensions of a few $\mathrm{cm}$. Reagents can then be brought together in a specific sequence, mixed and allowed to react for a specified time in a controlled region of the reactor channel network using (most commonly) electrokinetic (electroosmotic and electrophoretic) and/or hydrodynamic pumping.

For electrokinetically-driven systems, electrodes are placed in the appropriate reservoirs to which specific voltage sequences can be delivered under automated computer control. This control offers a simple but effective method of moving and separating reactants and products within a micro reactor, without the need for moving parts. Hydrodynamic pumping exploits conventional or micro-scale pumps, notably syringe-type pumps, to manoeuvre solutions around the channel network but has the disadvantage of requiring either large external pumps or complex fabrication of small moving parts. Detection of chemical species can be either 'on-chip', using, for example, microscopic imaging of absorbance or fluorescence signals or 'off-chip' by either reservoir sampling or suitable connection to a conventional benchtop instrument such as an HPLC or mass spectrometer. 
To date, the greatest research effort in the field of micro scale devices has been in the analytical arena. One of the main aims of this research is to develop a miniaturised total analytical system ( $\mu$-TAS). ${ }^{10-17}$ Optimally, such devices would automatically perform sampling, sample preparation, separation, detection and data processing in a fullyintegrated manner. In addition to the advantages of high, automated throughput and low reagent consumption with increased safety of operation resulting from low reagent quantities, these devices offer potential as remote controlled systems, which could be placed in inaccessible locations for continuous monitoring of chemical or environmental processes. To date, the most popular area of $\mu$-TAS research has been in the biomedical field, including the analysis of DNA and proteomics, ${ }^{18-25}$ and has resulted in the release of the first commercial analytical micro reactor device from Agilent, formerly Hewlett-Packard.

Alongside the continuing development of $\mu$-TAS and related analytical applications, a concerted effort has now begun to establish the benefits that micro reactors can bring to the field of reaction chemistry. The ability to manipulate reagent concentrations in both space and time by electrokinetic voltage control within the channel network of a micro reactor, provides an additional level of reaction control which is not attainable in bulk stirred reactors where concentrations are generally uniform. The spatial and temporal control of chemical reactions in micro reactors, coupled with the features of very small reaction volumes ( $\mu \mathrm{m}$ dimensions) and high surface interactions, is somewhat akin to the situation of reactions within biological cells. Nature exploits the organised distribution of reagents within the micron-sized sub-domains of cells to control and alter chemical reactivity relative to the situation of homogeneous solutions. Consistent with this notion, many reactions (as reviewed in Section 4) have been demonstrated to show altered reactivity, product yield and selectivity when performed in micro reactors as compared with conventional bench top glassware.

This review, covering key aspects of the fabrication and operation of micro reactors together with recent progress and potential applications in their use for chemical synthetic processes, is structured as follows. After this brief introduction, the fabrication of micro reactors is discussed in Section 2. Section 3 deals with the principles of electrokinetic operation of micro reactors and how the spatial and temporal evolution of reactions in micro reactors can be controlled. The range of liquid and gas phase reactions, including catalytic reactions, which have been performed in micro reactors is reviewed in Section 4. Potential applications and future directions are suggested in Section 5. Finally, conclusions and references are summarised.

\section{Fabrication and physical characterisation of micro reactors}

\subsection{Fabrication}

A number of materials such as silicon, quartz, glass, metals and polymers have been used to construct micro reactors. ${ }^{11}$ Important considerations in material choice include chemi- cal compatibility, ease and reproducibility of fabrication, whether or not the material supports electroosmotic flow (EOF) with the solvents of interest, and compatibility with detection methods. Glass is a popular choice since it allows EOF with many common solvents, is chemically inert, enables the use of visible light detection and fabrication methods are well established.

Depending on the material used, a range of channel microfabrication methods such as photolithography, hot embossing, powder blasting, injection moulding and laser micro forming are available. ${ }^{26}$ For glass micro reactors, photolithographic fabrication of channel networks is performed as shown schematically in Fig. 1 and described in Refs. 27, 28. Firstly, the channel network is designed and printed on a large scale using suitable computer drawing software. A film negative of the desired final size is then prepared by photoreduction to form the optical mask. Commerciallysupplied borosilicate glass photolithographic plates (thickness $3 \mathrm{~mm}$ ) coated with a thin metal etch mask layer (normally chromium) plus an upper layer of positive photoresist $(0.5-2.0 \mu \mathrm{m}$ depth) are used for channel network fabrication. The pattern of the required network of interconnecting channels is transferred from the optical mask to the photoresist layer. After light exposure, the photoresist is developed and removed, together with the chromium layer, to reveal the areas of glass to be etched. Recent instrumental advances of this process will, however, shortly

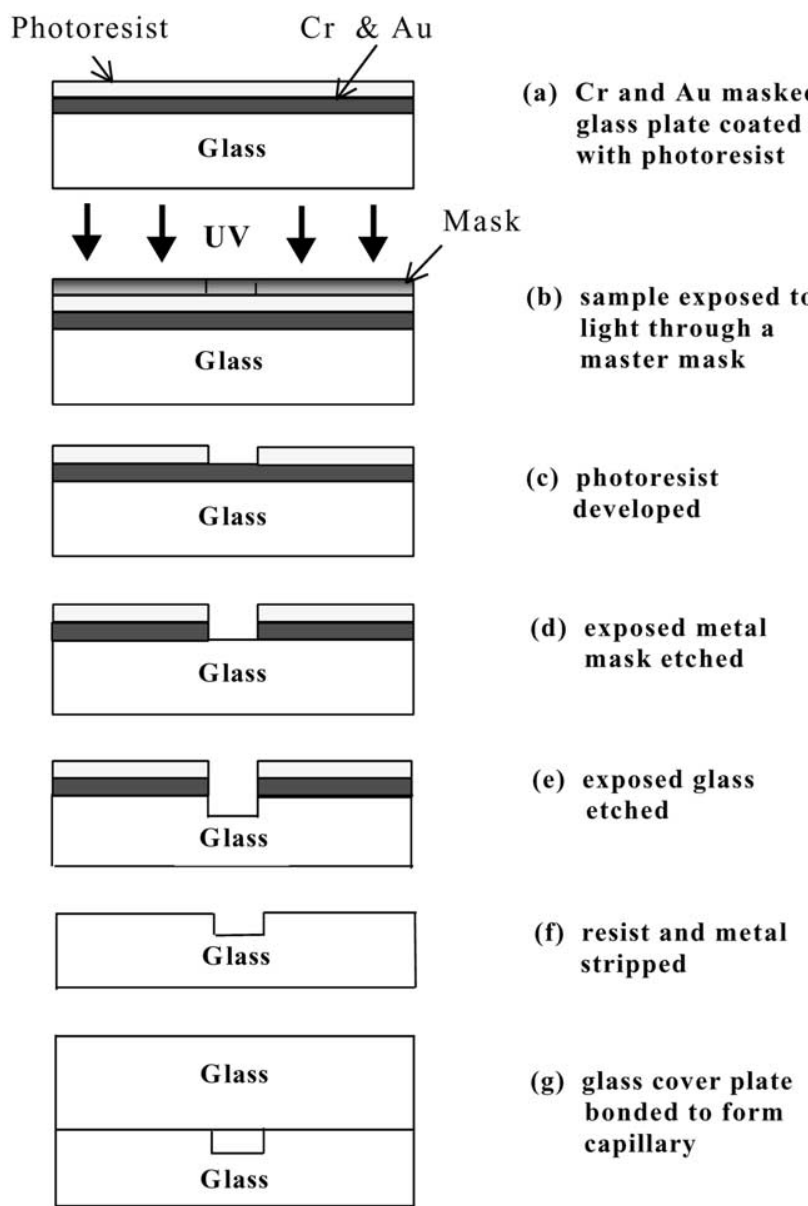

Figure 1. Photolithographic fabrication of channel networks in glass. 
enable the direct production of negatives from computer drawings. ${ }^{29}$ The patterned plate is then heated to allow the volatiles to evaporate, before performing the chemical etch. The channels are etched using a mixture of $1 \% \mathrm{HF}$ and 5\% $\mathrm{NH}_{4} \mathrm{~F}$ in water at $65^{\circ} \mathrm{C}$, resulting in an etch rate of $0.3-$ $0.5 \mu \mathrm{m} \mathrm{min}{ }^{-1}$. During etch it is important that the system is well agitated to ensure consistent supply of etchant to the surface plus removal of etch debris. In our laboratories, we have found that ultrasound irradiation during etch is effective.

In the procedure described above, glass is etched isotropically, i.e. the glass is etched away at equal rates in all directions from all points of exposed glass surface under the metal mask. The channel cross-sectional shape that results is shown in Fig. 2. For an initial optical mask channel width $m$ and an etch depth $d$, the channel cross-section consists of a rectangle of dimensions $(d \times m)$ flanked by two quarter circles of radius $d$ which undercut the mask. As shown in the micrograph of an exposed channel end, this theoretical channel shape is normally obtained in practice, although thermal bonding (discussed later) may result in some slight distortion. Note that, for isotropic etching, it is impossible to prepare channels the depth of which exceeds their width. Anisotropic materials, however, such as silicon, etch at different rates along different crystal planes. Etching of these materials therefore commonly produces V-shaped channels the geometry of which is related to the relative orientations of the etch mask and crystal planes, together with the differential etch rates. ${ }^{26}$

The base plate containing the etched channel network must next be sealed by bonding to a $17 \mathrm{~mm}$ upper plate contain-
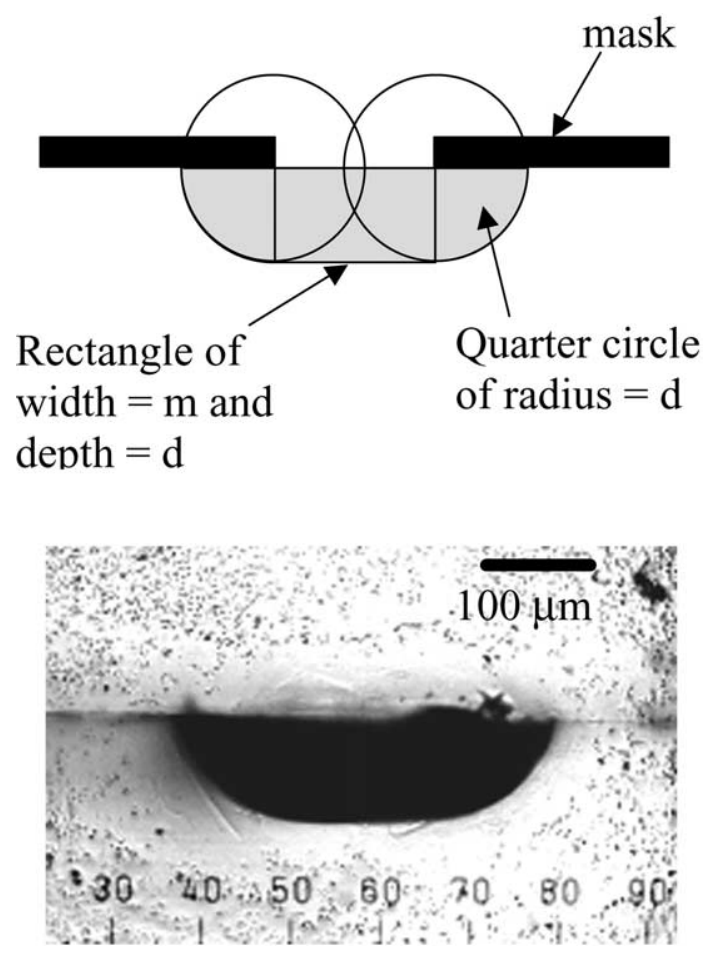

Figure 2. Diagram showing the relationship between mask width $m$, etch depth $d$ and the channel profile (grey shaded area) for an isotropic etch in glass. The micrograph shows a channel end view in a bonded glass micro reactor.

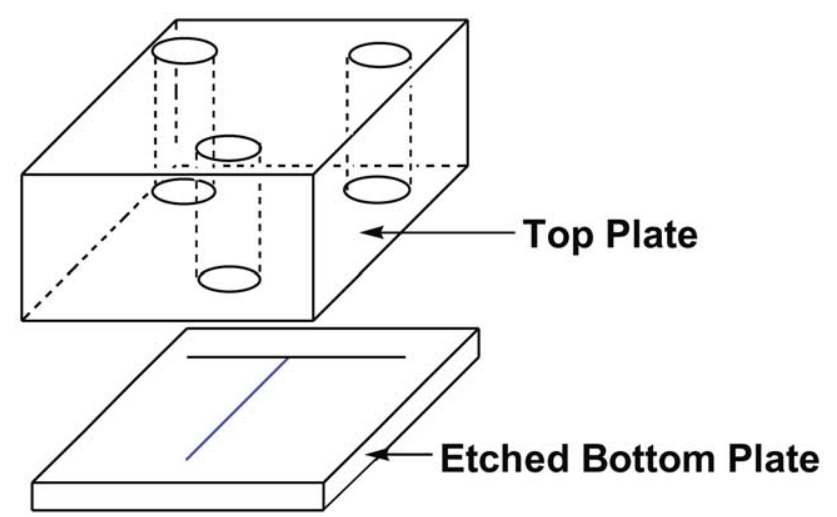

Figure 3. Exploded view of a micro reactor constructed from a base plate containing the etched channel network and thermally bonded to a thick upper plate containing reagent reservoirs.

ing pre-drilled holes to act as reservoirs for reagents. In our laboratories, the upper plate is aligned with the channel geometry and thermally bonded to the base plate (typically $575^{\circ} \mathrm{C}$ for $3 \mathrm{~h}$ ). ${ }^{27,28}$ Thermal bonding is aided by placing a weighting block of non-adhering quartz of high softening temperature on the upper plate. A schematic of an all-glass device produced by the method described is shown in Fig. 3 . For good thermal bonding, it is important to ensure that both the glass types of the upper and lower plates have the correct thermal softening and expansion properties. In addition, the surfaces to be bonded must be clean and flat.

\subsection{Determination of the 3D dimensions of the channel networks}

Having fabricated the micro reactor, it is essential to know the full set of dimensions in 3D of the channel network in order to establish volumetric flow rates and enable reaction characterisation. In the plane of the channel network, the lengths of the different channel sections are easily determined using optical microscopy. Channel profiles (depths and widths) can be determined using stylus profiling methods on the base plates before bonding. As mentioned earlier, however, thermal bonding can result in some degree of distortion of the channel profiles which can only be estimated by measurement of bonded devices. One approach for bonded chips is to cut the chip perpendicular to the channel axis followed by measurement of the channel profile using optical microscopy. This method is destructive and only enables the profile to be determined at a small number of pre-selected points. These disadvantages can be overcome by using a newly-developed method based on digital microscopic imaging of a dye solution-filled channel network. ${ }^{30}$ As shown in Fig. 4, the chip is mounted on a transmission microscope operating with monochromatic light at a wavelength corresponding to the maximum absorbance of a dye solution within the channel network. Using a digital camera on the microscope, $256 \times 256$ arrays of pixel light intensity values are recorded for the chip filled with dye solution $\left(I_{\text {dye }}\right)$, filled with solvent $\left(I_{\text {solvent }}\right)$ and 'dark' images $\left(I_{\text {dark }}\right)$. The data arrays are then converted to an array of absorbance values (Abs) using Eq. (1)

Abs $=\log _{10}\left(\frac{I_{\text {solvent }}-I_{\text {dark }}}{I_{\text {dye }}-I_{\text {dark }}}\right)$ 

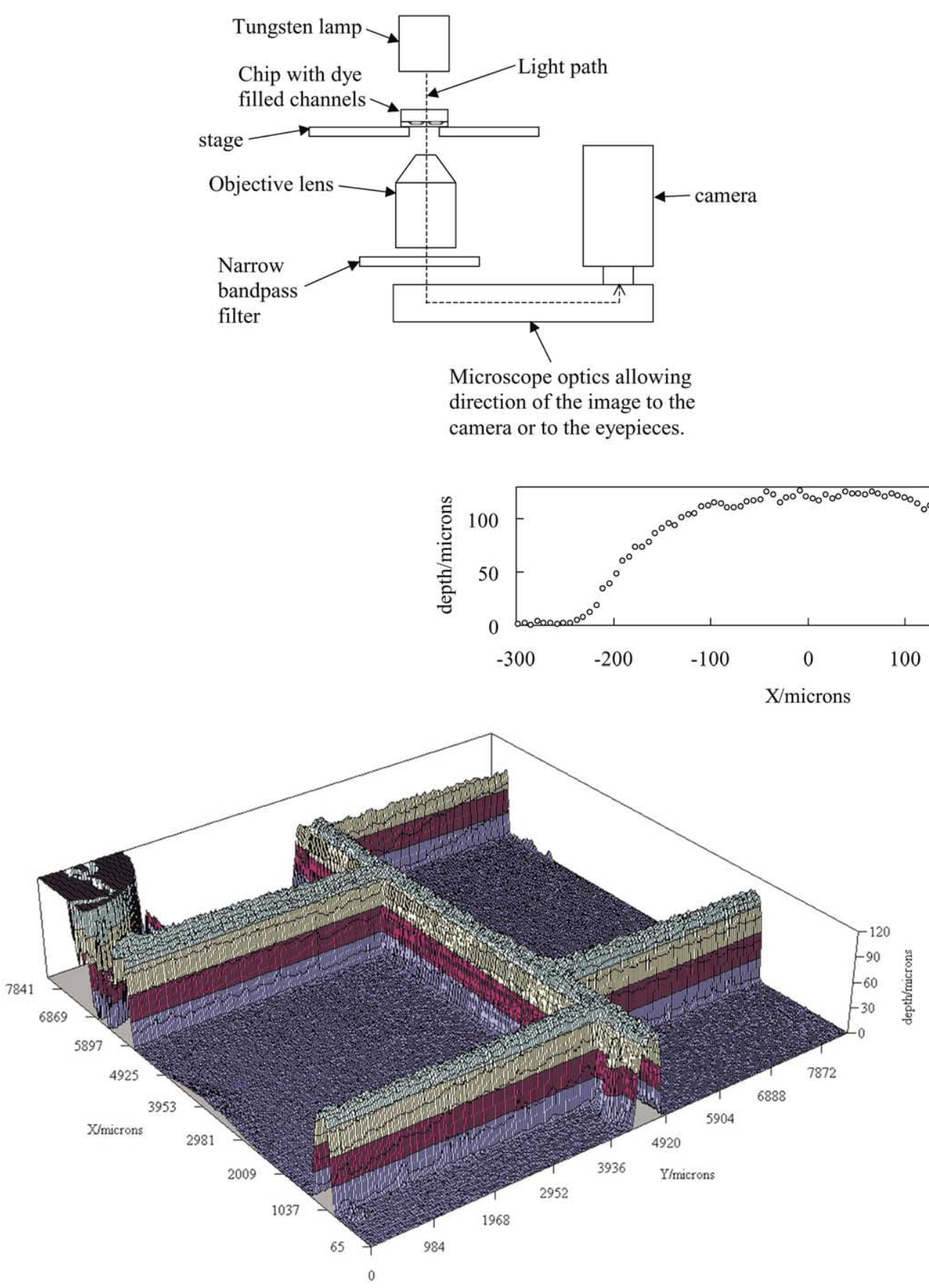

Figure 4. Digitised images taken using a transmission microscope can be used to produce full 3D profiles of a channel network. The inset plot shows a transverse slice across a channel.

Next by applying the Beer-Lambert Law Abs $=\varepsilon c l$ (where $\varepsilon$ is the extinction coefficient of the dye, $c$ is the dye concentration and $l$ is the optical path length), where the extinction coefficient $(\varepsilon)$ and concentration $(c)$ of the dye have been determined using a conventional UV/VIS spectrophotometer, the array of Abs values can be converted into an array of optical path-length values corresponding to the depth of each channel $(l)$, and the etched depth for all X-Y positions within the chip images are obtained. Fig. 4 shows a full $3 \mathrm{D}$ profile measured in this way for a section of a channel network. Analysis of 'slices' through the full array of data enables the determination of the cross- sectional channel profile within any part of the imaged network. Etched depths in the range from a few $\mu \mathrm{m}$ to hundreds of $\mu \mathrm{m}$ can be determined with a precision of a few percent. Having determined the path length $l$ for the channel network, the concentration profile of a coloured (or fluorescent) reactant or product species can be similarly derived from digital camera images, an example of this approach being illustrated in Section 3.3. As will become more apparent in the later sections, the physical geometry of a micro reactor (i.e. cross-sectional profiles and channel intersections) is intrinsically linked to the chemical processes it supports. The influence of surface-to-bulk 
effects, concentration (including $\mathrm{pH}$ ) and thermal gradients, for example, are all affected by the material and geometry of the micro reactor.

\section{Operation of micro reactors using electrokinetic control}

\subsection{Electroosmotic flow (EOF) and electrophoresis}

Pumping of solutions around a channel network by EOF using voltages applied via electrodes placed in the reservoirs has several significant advantages over alternative pumping methods. It can be easily miniaturised since no mechanical moving parts are involved and the required voltage sequences can be readily applied under automated computer control. For a glass micro reactor, the channel wall-solution interface normally has a negative charge, arising from ionisation of surface groups that are immobile. This immobile surface charge attracts a diffuse layer (of thickness of the order of $\mathrm{nm}$ ) of mobile, oppositely-charged counterions in the solution adjacent to the channel wall (cations for a negatively-charged glass channel wall). As shown schematically in Fig. 5, application of an electric field along the channel length causes the nm thick 'skin' of mobile cations to move towards the more negative electrode and drags all the intervening solution in the bulk of the channel with it. An important feature of EOF is that the liquid EOF velocity is constant across the channel except in the nm thick regions of the diffuse layer of counterions very close to the wall. Unlike EOF, pressure-driven flow produces a parabolic velocity profile with high velocities in the channel centre and slow velocities near to the wall, giving rise to increased 'blurring' of reagent zones along a channel length. Imaging of the different velocity profiles induced by EOF and pressure-driven flow has been described by Paul et al. ${ }^{31}$

The EOF fluid velocity $v_{\text {eof }}$ is given by Eq. $(2)^{32-34}$

$v_{\text {eof }}=\frac{E \varepsilon \varepsilon_{0} \zeta}{\eta}$

where $E$ is the electric field (voltage divided by electrode separation), $\varepsilon$ is the relative dielectric constant of the liquid, $\varepsilon_{0}$ is the permittivity of free space, $\zeta$ is the zeta potential of the channel wall-solution interface and $\eta$ is the liquid viscosity. For the glass-aqueous solution interface, the value of $\zeta$ varies from -50 to $-150 \mathrm{mV}$ at $\mathrm{pH} 7$ (dependent on ionic strength) but decreases to 0 at $\mathrm{pH}$ around $2.5{ }^{35}$ The magnitude of the electric field applied in micro reactors (typically some hundreds of $\mathrm{V} \mathrm{cm}^{-1}$ ) gives EOF velocities in the range $0.1-1 \mathrm{~mm} \mathrm{~s}^{-1}$ for aqueous solutions at $\mathrm{pH}$.

From Eq. (2), it can be seen that $v_{\text {eof }}$ is proportional to the applied voltage and depends on the properties of both the liquid and the channel material. EOF is not obtained with semiconductor materials such as silicon and does not occur with low polarity solvents such as alkanes where no diffuse layer of surface counterions exists. The EOF velocity $v_{\text {eof }}$ is independent of the channel cross-sectional dimensions whereas the EOF volumetric flow rate (given by the product of $v_{\text {eof }}$ multiplied by the channel cross-sectional area) does depend on the channel dimensions. Additionally, it is important to note that the channel dimensions determine whether 'pure' EOF or a mixture of EOF and pressure-driven flow is actually obtained in practice. As indicated above, flow within a micro reactor produces liquid height differences between the reagent reservoirs which, in turn, may produce pressure-driven flow opposing the EOF and disturbing the flat velocity profile for pure EOF. As discussed in Ref. 36, pressure-driven flow arising from small $(\mathrm{mm})$ reservoir height differences can be significant for relatively large channels but is suppressed for small channels where the hydrodynamic resistance is large. Overall, the channel dimensions must be designed to be sufficiently small such that pressure-driven flows are negligibly small relative to the EOF and errors arising from variable, pressure-driven flows (dependent on the reservoir liquid heights) are absent.

Under EOF voltage control, the solvent and any uncharged solutes move with a velocity $v_{\text {eof }}$. Within the electric field, charged solutes have an additional electrophoretic velocity $v_{\text {ph }}$ which is given by Eq. $(3)^{32,34}$

$v_{\mathrm{ph}}=\frac{z e E D}{k T}$

where $z$ is the charge number on the species, $e$ is the electronic charge, $D$ is the diffusion coefficient of the species, $k$ is the Boltzmann constant and $T$ is the absolute temperature. The magnitude of $v_{\mathrm{ph}}$ for typical micro reactor operating voltages is commonly comparable with $v_{\text {eof. }}$. The total velocity of a charged species is given by the vector sum of $v_{\text {eof }}$
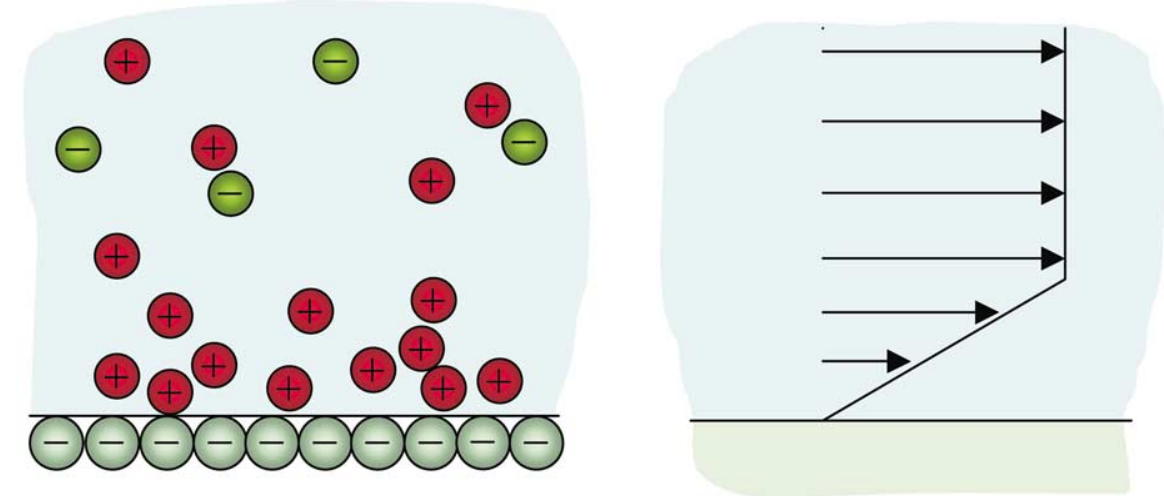

Figure 5. Voltage-driven movement of the diffuse layer of cations adsorbed at the negatively charged channel wall (left-hand figure) produces a flat EOF velocity profile across the channel except within the $\mathrm{nm}$ thick diffuse counterion layer (right-hand figure). 
and $v_{\mathrm{ph}}$. As noted above, the direction of EOF flow for aqueous solutions in a glass micro reactor is normally towards the more negative electrode. For a cationic solute species, $v_{\text {ph }}$ will therefore be in the same direction as the EOF (i.e. towards the more negative electrode) and will move faster down the channel than the solvent and neutral species. Anionic solutes will be retarded and may, if the magnitude of $v_{\mathrm{ph}}$ is greater than $v_{\text {eof }}$, move in the opposite direction. In this way, electrophoretic separation of solutes occurs along with EOF in micro reactor channels when operated using electrokinetic control. The ability to use electrophoretic mobility to move charged reagents or species and products within a micro reactor independently of the solvent has been exploited in micro reactors and brings a rather novel concept to reaction chemistry. In the Wittig chemistry described in Section 4, for example, the charged base and phosphonium salt used to form the intermediate ylide move and mix rapidly by electrophoresis, negating the need to move and mix the solvent. In addition, in reactions where separation of the products and reactants in real time is advantageous, electrophoretic mobility is a distinct advantage. Differential electrophoretic mobilities have been exploited to selectively control product detection times in the capillary electrophoresis technique of electrophoretically-mediated microanalysis (EMMA). ${ }^{37-40}$

\subsection{Control of reagent mobility in a channel network}

The strategy of using micro reactors to electrokinetically control the spatial and temporal evolution of chemical reactions relies on the ability to use voltage sequences to direct reagents to selected points at specified times within a channel network. For an intricate channel network with many reservoirs and electrodes, the relationships between the applied voltages and the liquid flow rates and solute mobilities in the different sections of the network can be very complex. The basic approach is to analyse the channel network in terms of an equivalent DC circuit where the different channel sections correspond to resistance elements and, as will be shown, the electrical currents are proportional to the volumetric liquid flow rates within the corresponding channel sections. ${ }^{36,41}$ As an illustrative example, we discuss the channel network shown in Fig. 6 where we wish to achieve a flow-inject-flow sequence to obtain a 'slug' of one reagent in a flowing stream of a second reagent. In the 'flow' mode, we require flow from reservoir A to D with zero flow from both B and C to D. In the 'inject' mode, we require flow from $\mathrm{B}$ to $\mathrm{C}$ with zero flow from $\mathrm{A}$ to D. To obtain both modes, a multichannel voltage supply operating under computer control using LabVIEW software is used to deliver the correct voltage sequences to the electrodes in reservoirs $\mathrm{A}, \mathrm{B}$ and $\mathrm{C}$ (voltages $V_{\mathrm{A}}, V_{\mathrm{B}}$ and $V_{\mathrm{C}}$ ) relative to reservoir $\mathrm{D}$, set to ground voltage. The control system used at Hull also enables computer logging of all voltages and currents during a run. The following analysis shows how the voltages are related to the electrical currents $I_{\mathrm{A}}, I_{\mathrm{B}}$ and $I_{\mathrm{C}}$ which, in turn, are related to the liquid volumetric flow rates in the corresponding channel sections $F_{\mathrm{A}}$, $F_{\mathrm{B}}$ and $F_{\mathrm{C}}$.

We consider the simplest possible situation in which the channel network of Fig. 6 is filled with a liquid of uniform electrical conductivity $\kappa$ and the zeta potential of the
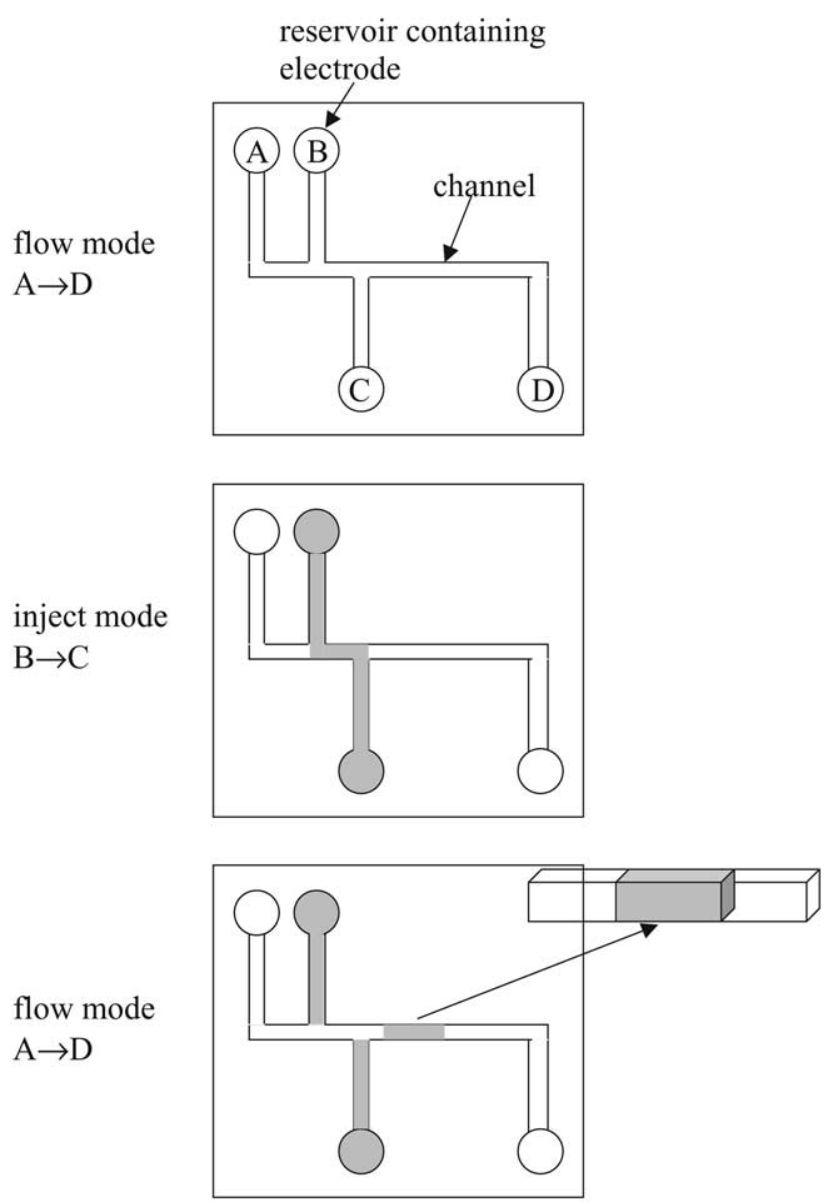

Figure 6. Double T-junction channel network and flow-inject-flow required to achieve a slug of one reagent within a flowing stream of a second reagent.

channel wall is equal in all channel sections. Experimentally, this situation can be achieved even when mixing different reactant solutions by ensuring a high background ('swamping') concentration of inert electrolyte in the different reagent solutions to be mixed. For the sake of simplicity

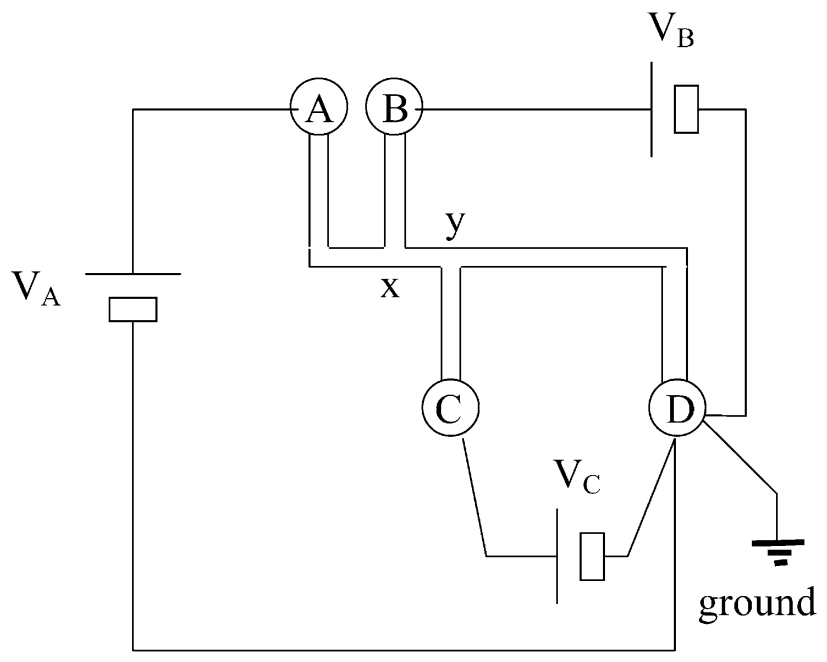

Figure 7. DC circuit used to drive the channel network of Fig. 6. Reservoir voltages (relative to ground in $\mathrm{D}$ ) are $V_{\mathrm{A}}, V_{\mathrm{B}}$ and $\mathrm{x}$ and y denote the channel junctions. The electrical current in channel section $\mathrm{Ax}$ is $I_{\mathrm{A}}$, section $\mathrm{Bx}$ is $I_{\mathrm{B}}$, section Cy is $I_{\mathrm{C}}$, section xy id $\left(I_{\mathrm{A}}+I_{\mathrm{B}}\right)$ and section yD is $\left(I_{\mathrm{A}}+I_{\mathrm{B}}+I_{\mathrm{C}}\right)$. 
we ignore the (normally small) contribution to the overall conductivity of a channel section which arises from surface conduction. ${ }^{32-34,41}$ With these approximations, the electrical resistance of the $i$ th channel section $R_{i}$ is given by Eq. (4)

$R_{i}=\frac{L_{i}}{\kappa A_{i}}$

where $L_{i}$ and $A_{i}$ are the length and cross-sectional area of the $i$ th channel section. The DC circuit used to drive flow in the channel network of Fig. 6 is shown in Fig. 7 and serves to define the current values in the different channel sections. In each of the limbs of the coupled circuit, the applied voltages are equal to the sum of the products of the resistances and currents and one ends up with a series of $(n-1)$ simultaneous equations where $n$ is the number of reservoirs containing electrodes (it is $n-1$ since one electrode is set to ground). For the channel network considered here, we have the three equations incorporated in Eq. (5)

$V_{\mathrm{A}}=R_{\mathrm{Ax}} I_{\mathrm{A}}+R_{\mathrm{xy}}\left(I_{\mathrm{A}}+I_{\mathrm{B}}\right)+R_{\mathrm{yD}}\left(I_{\mathrm{A}}+I_{\mathrm{B}}+I_{\mathrm{C}}\right)$

$V_{\mathrm{B}}=R_{\mathrm{Bx}} I_{\mathrm{B}}+R_{\mathrm{xy}}\left(I_{\mathrm{A}}+I_{\mathrm{B}}\right)+R_{\mathrm{yD}}\left(I_{\mathrm{A}}+I_{\mathrm{B}}+I_{\mathrm{C}}\right)$

$V_{\mathrm{C}}=R_{\mathrm{Cy}} I_{\mathrm{C}}+R_{\mathrm{yD}}\left(I_{\mathrm{A}}+I_{\mathrm{B}}+I_{\mathrm{C}}\right)$

where $R_{i}$ is the resistance of the $i$ th channel section. Solving these simultaneous equations (achieved most easily using a software package such as MathCAD) yields the corresponding expressions for the electrical currents shown in Eq. (6)

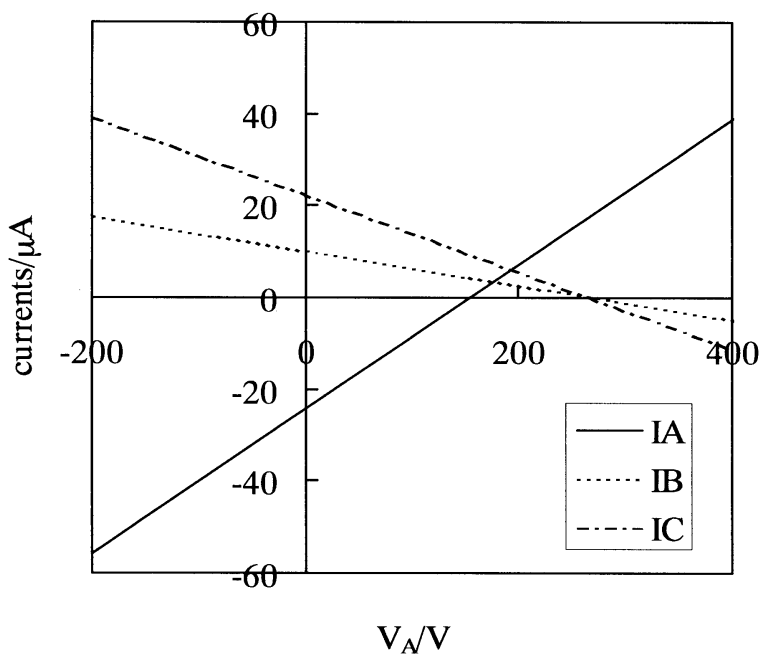

Figure 8. Illustrative plots of electrical currents versus $V_{\mathrm{A}}$ for $V_{\mathrm{B}}$ and $V_{\mathrm{C}}$ both set to $200 \mathrm{~V}$. The data refer to the channel network of Fig. 6 filled with $50 \mathrm{vol} \%$ ethanol/water containing $35 \mathrm{mM}$ phosphate buffer at $\mathrm{pH}$ 7.5.

Representative plots of electrical currents versus one of the voltages (with the remaining voltages being fixed) are shown in Fig. 8. The current values are typically in the $\mu \mathrm{A}$ range and, as expected from Ohm's law, they are proportional to the voltage. However, because of the linking together of the different limbs of the overall circuit, the

$$
\begin{gathered}
I_{\mathrm{A}}=\frac{\left(R_{\mathrm{Cy}} R_{\mathrm{xy}}+R_{\mathrm{Cy}} R_{\mathrm{yD}}+R_{\mathrm{Bx}} R_{\mathrm{yD}}+R_{\mathrm{xy}} R_{\mathrm{yD}}+R_{\mathrm{Cy}} R_{\mathrm{Bx}}\right) V_{\mathrm{A}}-\left(R_{\mathrm{Cy}} R_{\mathrm{yD}}+R_{\mathrm{Cy}} R_{\mathrm{xy}}+R_{\mathrm{xy}} R_{\mathrm{yD}}\right) V_{\mathrm{B}}-R_{\mathrm{Bx}} R_{\mathrm{yD}} V_{\mathrm{C}}}{R_{\mathrm{Cy}} R_{\mathrm{Bx}} R_{\mathrm{Ax}}+R_{\mathrm{Cy}} R_{\mathrm{xy}} R_{\mathrm{Bx}}+R_{\mathrm{Cy}} R_{\mathrm{xy}} R_{\mathrm{Ax}}+R_{\mathrm{Cy}} R_{\mathrm{yD}} R_{\mathrm{Bx}}+R_{\mathrm{Cy}} R_{\mathrm{yD}} R_{\mathrm{Ax}}+R_{\mathrm{Bx}} R_{\mathrm{yD}} R_{\mathrm{Ax}}+R_{\mathrm{xy}} R_{\mathrm{yD}} R_{\mathrm{Bx}}+R_{\mathrm{xy}} R_{\mathrm{yD}} R_{\mathrm{Ax}}} \\
I_{\mathrm{B}}=\frac{-\left(R_{\mathrm{Cy}} R_{\mathrm{xy}}+R_{\mathrm{Cy}} R_{\mathrm{yD}}+R_{\mathrm{Bx}} R_{\mathrm{yD}}\right) V_{\mathrm{A}}-\left(R_{\mathrm{Cy}} R_{\mathrm{xy}}+R_{\mathrm{Cy}} R_{\mathrm{yD}}+R_{\mathrm{Cy}} R_{\mathrm{Ax}}+R_{\mathrm{xy}} R_{\mathrm{yD}}+R_{\mathrm{yD}} R_{\mathrm{Ax}}\right) V_{\mathrm{B}}-R_{\mathrm{Ax}} R_{\mathrm{yD}} V_{\mathrm{C}}}{R_{\mathrm{Cy}} R_{\mathrm{Bx}} R_{\mathrm{Ax}}+R_{\mathrm{Cy}} R_{\mathrm{xy}} R_{\mathrm{Bx}}+R_{\mathrm{Cy}} R_{\mathrm{xy}} R_{\mathrm{Ax}}+R_{\mathrm{Cy}} R_{\mathrm{yD}} R_{\mathrm{Bx}}+R_{\mathrm{Cy}} R_{\mathrm{yD}} R_{\mathrm{Ax}}+R_{\mathrm{Bx}} R_{\mathrm{yD}} R_{\mathrm{Ax}}+R_{\mathrm{xy}} R_{\mathrm{yD}} R_{\mathrm{Bx}}+R_{\mathrm{xy}} R_{\mathrm{yD}} R_{\mathrm{Ax}}} \\
I_{\mathrm{C}}=\frac{-\left(R_{\mathrm{Bx}} R_{\mathrm{yD}}\right) V_{\mathrm{A}}-\left(R_{\mathrm{yD}} R_{\mathrm{Ax}}\right) V_{\mathrm{B}}-\left(R_{\mathrm{Bx}} R_{\mathrm{Ax}}+R_{\mathrm{yD}} R_{\mathrm{Ax}}+R_{\mathrm{xy}} R_{\mathrm{Ax}}+R_{\mathrm{xy}} R_{\mathrm{Bx}}+R_{\mathrm{Bx}} R_{\mathrm{yD}}\right) V_{\mathrm{C}}}{R_{\mathrm{Cy}} R_{\mathrm{Bx}} R_{\mathrm{Ax}}+R_{\mathrm{Cy}} R_{\mathrm{xy}} R_{\mathrm{Bx}}+R_{\mathrm{Cy}} R_{\mathrm{xy}} R_{\mathrm{Ax}}+R_{\mathrm{Cy}} R_{\mathrm{yD}} R_{\mathrm{Bx}}+R_{\mathrm{Cy}} R_{\mathrm{yD}} R_{\mathrm{Ax}}+R_{\mathrm{Bx}} R_{\mathrm{yD}} R_{\mathrm{Ax}}+R_{\mathrm{xy}} R_{\mathrm{yD}} R_{\mathrm{Bx}}+R_{\mathrm{xy}} R_{\mathrm{yD}} R_{\mathrm{Ax}}}
\end{gathered}
$$

The volumetric liquid flow rate in the $i$ th channel section $F_{i}$ is proportional to the corresponding electrical current $I_{i}$ according to Eq. (7), ${ }^{32-34}$

$F_{i}=-I_{i}\left(\frac{\varepsilon \varepsilon_{0} \zeta}{\eta \kappa}\right)$

In order to determine the voltages required to achieve a particular pattern of flow rates (e.g. flow or inject modes described above), all the resistance values of the different channel sections, filled with the liquid of interest, must be known. This can be done by measuring the currents as a function of the various voltages within the micro reactor and computer fitting the resistance values. Alternatively, one can measure the liquid conductivity (using a conventional conductivity meter) and all the channel dimensions and calculate the required resistances according to Eq. (4). Calibration measurements of liquid flow rates within the micro reactor as a function of different set currents yield the value of $\zeta$ and then enable the conversion of measured currents (logged during a run) to liquid flow rates from Eq. (7).

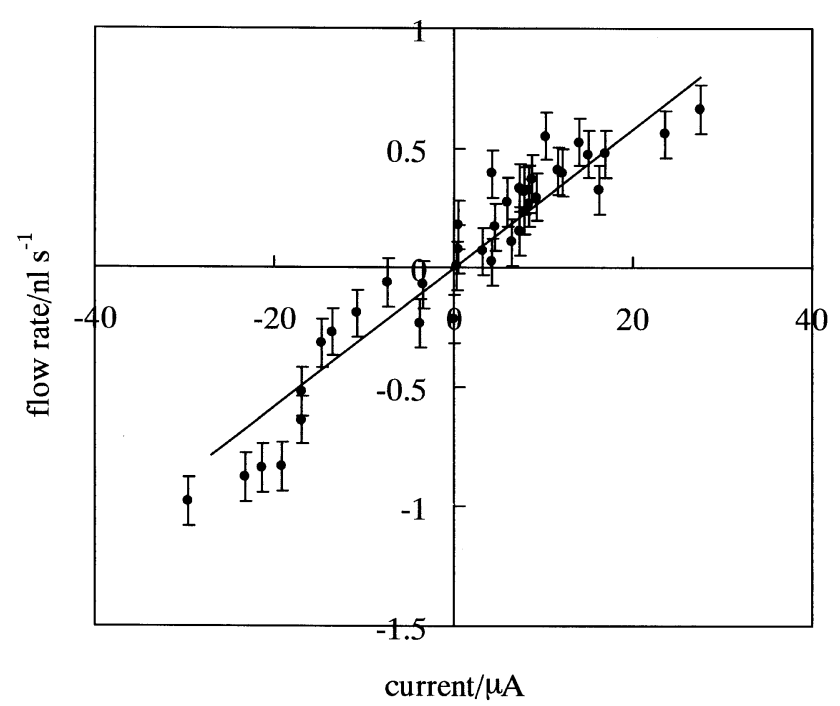

Figure 9. Correlation between measured volumetric liquid flow rates and the corresponding electrical currents for the channel network of Fig. 6 filled with $50 \mathrm{vol} \%$ ethanol/water containing $35 \mathrm{mM}$ phosphate buffer at $\mathrm{pH}$ 7.5. 
Table 1. Voltages, electrical currents, volumetric flow rates and liquid EOF velocities for flow and injection modes in the system of Fig. 9

\begin{tabular}{lcc}
\hline Variable & Flow mode $(\mathrm{A} \rightarrow \mathrm{D})$ & Inject mode $(\mathrm{B} \rightarrow \mathrm{C})$ \\
\hline$V_{\mathrm{A}}(\mathrm{V})$ & 225 & 1 \\
$V_{\mathrm{B}}(\mathrm{V})$ & 169 & 86 \\
$V_{\mathrm{C}}(\mathrm{V})$ & 169 & -38 \\
$I_{\mathrm{A}}(\mu \mathrm{A})$ & $15(\mathrm{set})$ & $0(\mathrm{set})$ \\
$I_{\mathrm{B}}(\mu \mathrm{A})$ & $0(\mathrm{set})$ & $8(\mathrm{set})$ \\
$I_{\mathrm{C}}(\mu \mathrm{A})$ & $0(\mathrm{set})$ & $-8(\mathrm{set})$ \\
$\left.F_{\mathrm{A}}(\mathrm{nl} \mathrm{s})^{-1}\right)$ & 0.45 & 0 \\
$\left.F_{\mathrm{B}}(\mathrm{nl} \mathrm{s})^{-1}\right)$ & 0 & 0.24 \\
$\left.F_{\mathrm{C}}(\mathrm{nl} \mathrm{s})^{-1}\right)$ & 0 & -0.24 \\
$v_{\text {eof }}(\mathrm{A})\left(\mathrm{mm} \mathrm{s}^{-1}\right)$ & 0.05 & 0 \\
$v_{\text {eof }}(\mathrm{B})\left(\mathrm{mm} \mathrm{s}^{-1}\right)$ & 0 & 0.03 \\
$v_{\text {eof }}(\mathrm{C})\left(\mathrm{mm} \mathrm{s}^{-1}\right)$ & 0 & -0.03 \\
\hline
\end{tabular}

voltages required to produce zero current in a particular channel section are determined by a complex function of all resistance and voltage values. An example of the linear correlation between the electrical current and liquid flow rates in corresponding channel sections is shown in Fig. 9. Applying this type of information, Table 1 lists the calculated voltage settings required to achieve flow and injection modes with set flow rates for the system shown in Fig. 9.

Full details of this entire procedure are described in Ref. 41 where it is demonstrated that measurement of the liquid conductivity $\kappa$, zeta potential $\zeta$ and all channel dimensions enables the correct calculation of the voltages needed to achieve any set of flow rates.

Several notes of caution about this analysis should be emphasised. Firstly, the study described in Ref. 41 indicates that correction of the overall electrical current for surface conduction along the channel walls is sometimes required. The surface conduction may become non-negligible when the bulk conductivity of the liquid is relatively low. Secondly, the analysis given here applies to the situation when the liquid conductivity and the zeta potential are uniform throughout the channel network. Mixing of reagent solutions which possess different conductivities and/or which have different zeta potentials with the channel walls

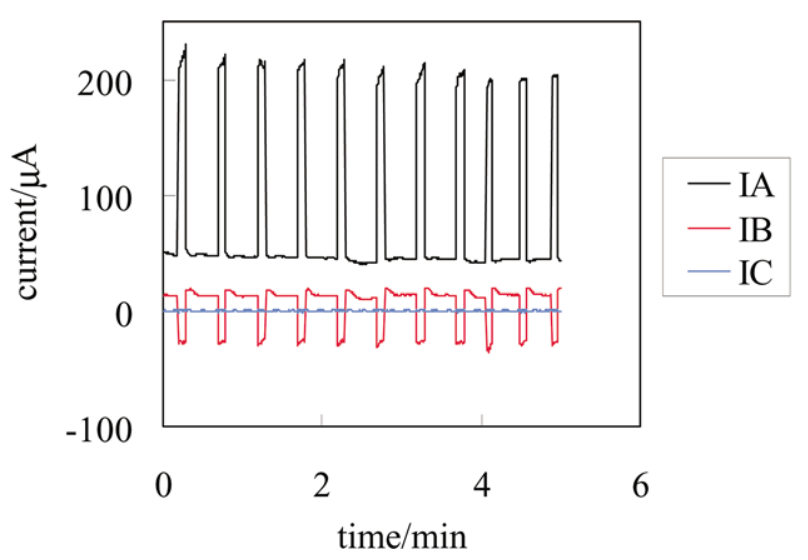

Figure 10. Electrical current oscillations due to electrolytic formation and subsequent dissolution of gas bubbles in the micro reactor channel network of Fig. 6. will produce moving channel zones with non-uniform properties and EOF mobilities. Obviously, this situation requires a considerably more complex analysis. Lastly, the simplified analysis presented here, although sufficient to illustrate the main features of flow control, takes no account of flow inertial effects and the non-uniformity of the electrical field around channel junctions. At a microscopic level, the effects cause complex local flow patterns in these regions, which can be modelled using sophisticated fluid dynamics software. Interested readers are referred to Refs. 42-46 for progress in this field.

In addition to its use in determining the required electrokinetic flow control parameters, the routine, in situ monitoring of electrical currents in channel networks provides useful diagnostic information on micro reactor operation. Firstly, the observation of a current dropping to zero in a branch of the channel network can indicate the position of a channel blockage. Secondly, application of an excessive voltage across a channel network can cause electrochemical reactions that may result in the release of gas bubbles. Fig. 10 shows an example of a current trace where a series of regular 'spikes' were caused by the liberation and subsequent dissolution of hydrogen bubbles at an electrode. Electrolysis occurs in micro reactors when the overall voltage difference between an electrode pair (typically several hundred V) is sufficiently high or the electrical resistance of the relevant channel section is low such that the voltage change across an electrode/solution interface (typically a fraction of a $\mathrm{V}$ ) exceeds the redox potential required for an electrochemical reaction. Lastly, when chemical reactions occur in a micro reactor and result in a change of conductivity of the solution, in situ current monitoring can, in principle, be exploited to monitor the extent of a reaction within the channel network. Fig. 11 shows the change in conductivity observed during the progress of a Wittig reaction, where methanol is used as the solvent.

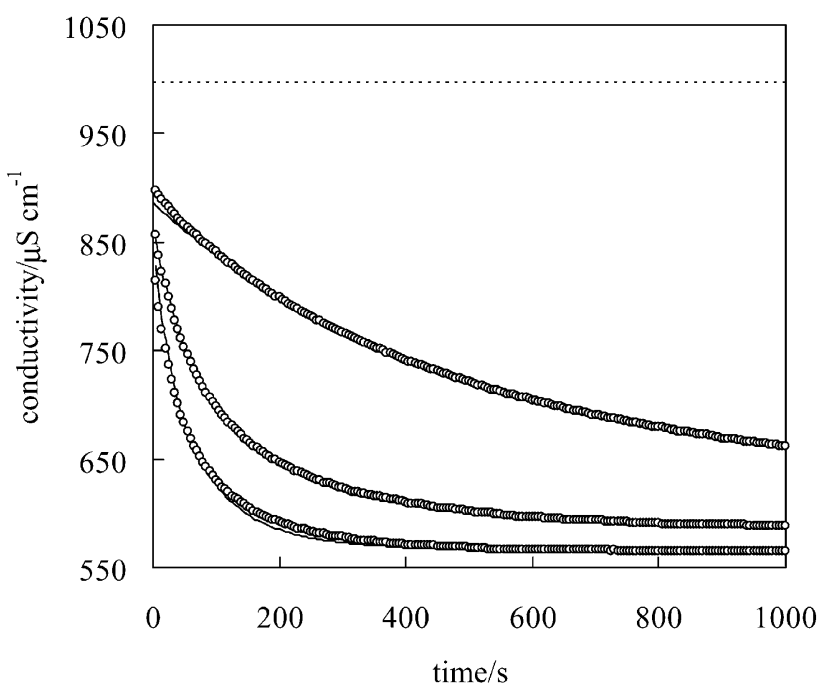

Figure 11. Conductivity changes which occur during the Witting reaction of 2-nitrophenylphonium bromide (NTPB) $(5 \mathrm{mM})$, sodium methoxide base $(7.5 \mathrm{mM})$ and 0,15 , and $30 \mathrm{mM}$ methyl 4-formylbenzaldehyde (MFB) in methanol at $20^{\circ} \mathrm{C}$. The reaction proceeds via the fast reversible formation of the ylid which then either decomposes or reacts to form an adduct with MFB. The horizontal line shows the conductivity of the reagents in the absence of the formation of the ylid intermediate. 


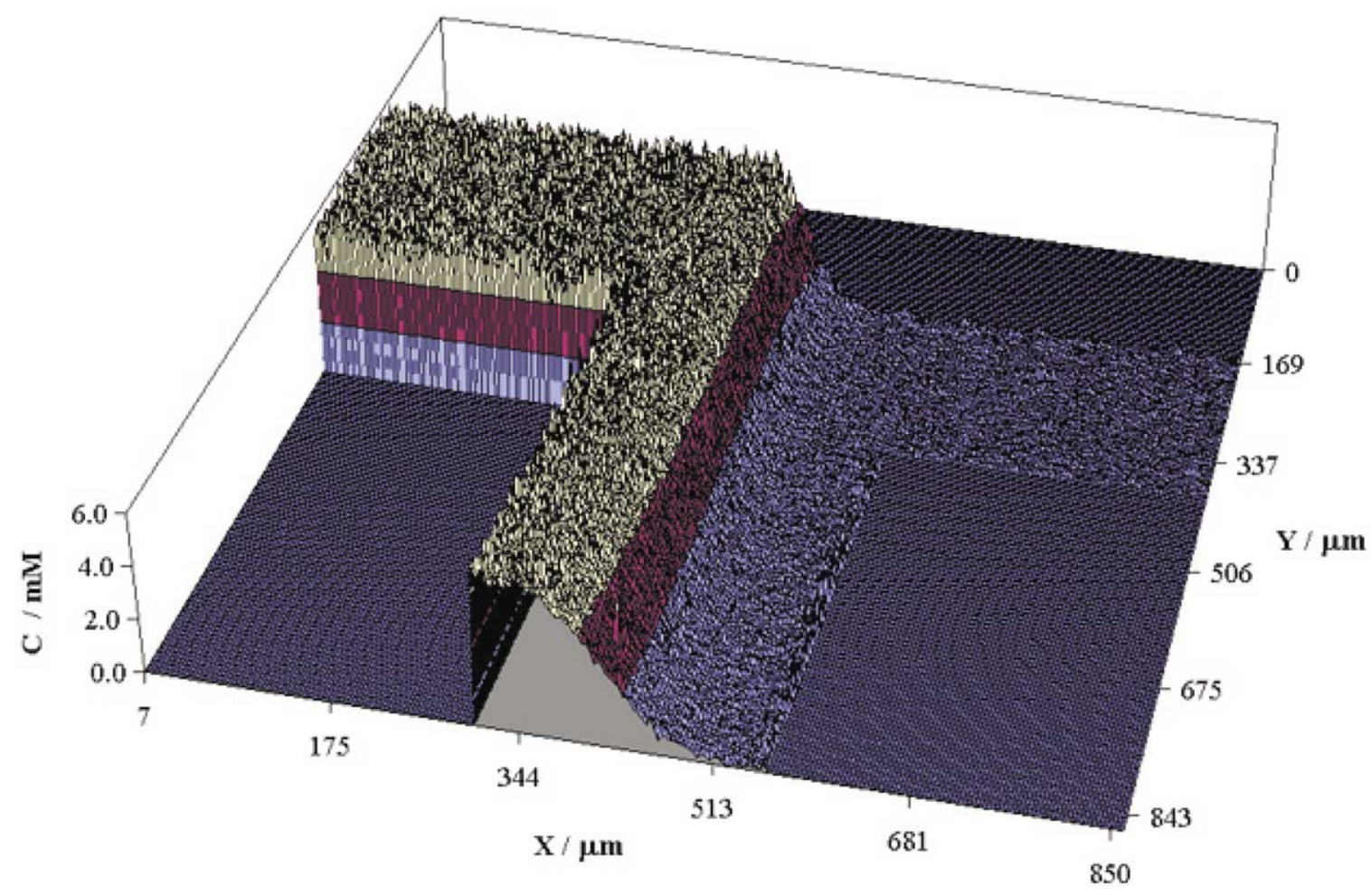

Figure 12. 3D plot of dye concentration versus position within a T-junction. The dye solution enters from the left and is combined with solvent entering from the right. The flow velocity down the 'leg' of the $\mathrm{T}$ is $0.48 \mathrm{~mm} \mathrm{~s}^{-1}$. Detailed analysis of the diffusional broadening of the dye-solvent interface seen as the laminar streams move down the channel (corresponding to different tomes after contact) yields the diffusion coefficient of the dye $\left(1.0 \times 10^{-10} \mathrm{~m}^{2} \mathrm{~s}^{-1}\right.$ in this example).

\subsection{Reagent mixing and chemical reaction in a micro reactor}

Except for unimolecular reactions, two or more reactant solutions must be mixed as a necessary first step to achieving reaction. Two main modes of mixing are possible which have very different characteristics. The first mode, denoted 'diffusive mixing', is illustrated in Fig. 12 which shows the $3 \mathrm{D}$ concentration profile resulting when a dye solution (entering from the left channel) is combined with solvent entering from the right-hand channel of a T-junction. The two solutions flow together down the leg of the T. For typical EOF conditions, the linear fluid velocities are of the order of $0.1-1 \mathrm{~mm} \mathrm{~s}^{-1}$. The Reynolds number $R_{\mathrm{e}}$ (equal to $D_{\mathrm{e}} v \rho / \eta$ where $D_{\mathrm{e}}$ is the effective channel diameter, $v$ is the velocity, $\rho$ is the liquid density and $\eta$ is the liquid viscosity) for such flow is $<10$, well below the transition from laminar to turbulent flow which occurs at $R_{\mathrm{e}}$ values of around 2500. The co-flowing reagent streams therefore retain their laminar flow pattern and mixing occurs only by inter-diffusion. From the $3 \mathrm{D}$ concentration plot of the dye, it can be seen that only a small extent of diffusional mixing occurs in the time between the first contact of the solution streams' (at the top edge of the image) flow to a position corresponding to the bottom edge of the image. For the flow velocity used $\left(0.48 \mathrm{~mm} \mathrm{~s}^{-1}\right)$, this time corresponds to approximately $2 \mathrm{~s}$. In this mode of mixing, at very long times the reagent concentrations are diluted by a ratio equal to the ratio of the flow rates of the incoming streams. A crude estimate of the time required for mixing across the complete width of the channel (w) is given by $t \approx 5 w^{2} / D$, where $D$ is the diffusion coefficient of the solute species.
For a typical channel width of $100 \mu \mathrm{m}$ and a value of $D$ of $5 \times 10^{-10} \mathrm{~m}^{2} \mathrm{~s}^{-1}$, this time is of the order of $100 \mathrm{~s}$. It can be seen that the mixing of two streams flowing at $1 \mathrm{~mm} \mathrm{~s}^{-1}$ in a typical channel length of a few $\mathrm{cm}$ will normally be very incomplete. Imaging of the diffusive broadening of the concentration gradient perpendicular to the laminar-flowing streams at different times has in fact been used to determine diffusion coefficients of absorbing or fluorescent species. ${ }^{47}$

Complete mixing by diffusion can be achieved by using slow (or stopped) flow to increase the contact time. Alternatively, faster flow or smaller channel cross-sections, within long channels (generally folded to reduce overall device size), will achieve the same result. Elegant designs of networks containing cascades of $\mathrm{T}$-junctions connected by suitably long, tortuous channels have been used to produce output channels giving simultaneously a series of fully-mixed dilutions of the original input solution. ${ }^{48,49}$ This type of multiple output is useful when using a micro reactor to determine, for example, a calibration plot of detector signal versus the concentration of an analyte species. The fact that the laminar flows of reagent streams inter-diffuse only relatively slowly has been exploited by using chemically-reactive reagent streams. Reactions between appropriate reagents at the interface between laminar, co-flowing streams of the reactants have been used, for example, to deposit very thin lines of metals (plus a range of other species) at the channel base as a novel means of fabricating 'nanowires' in specified locations. ${ }^{50}$

The second mode of mixing, 'slug injection', is illustrated in Fig. 6. Using a pair of offset T-junctions and the 


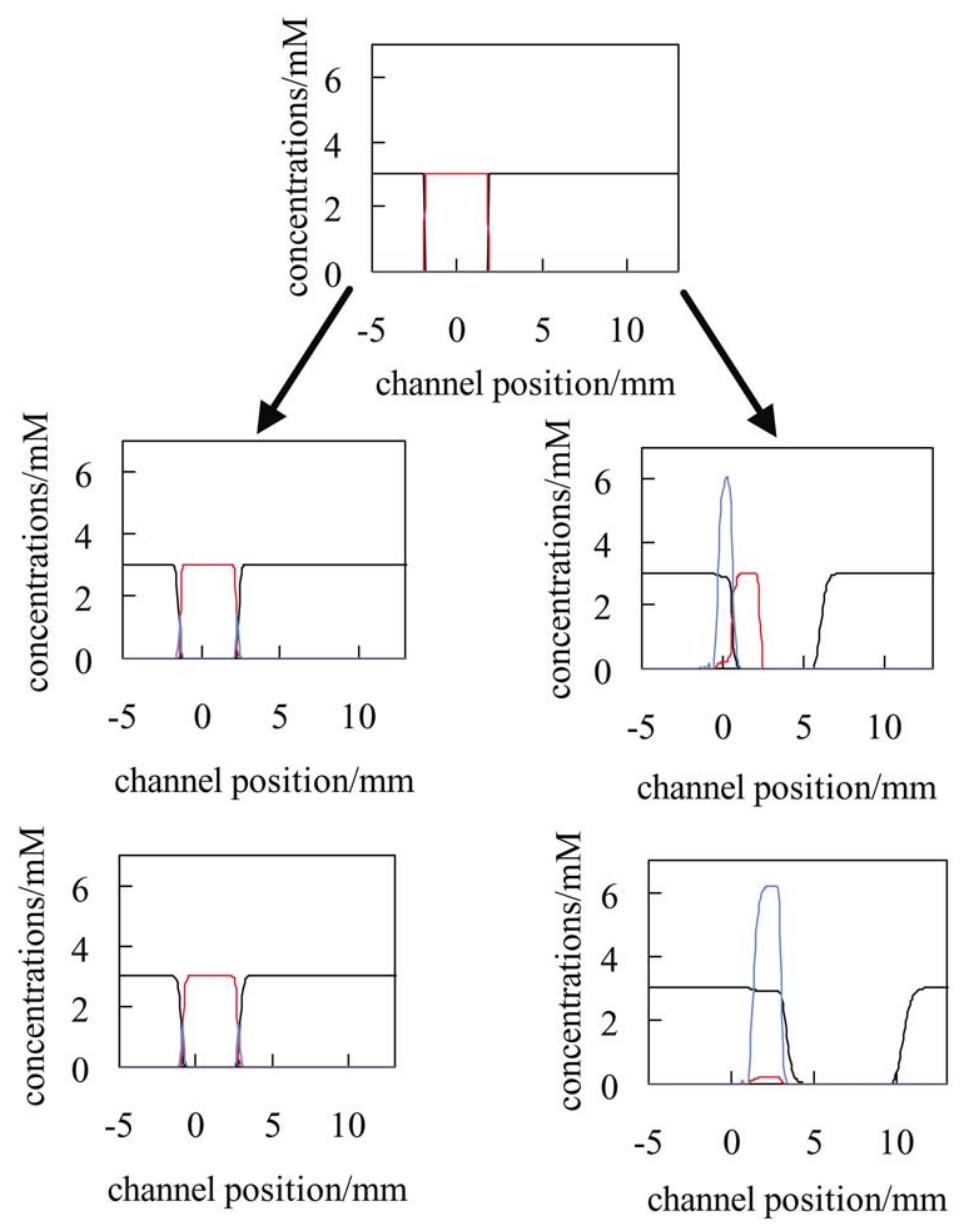

Figure 13. Time snapshots at 0,10 and 20 s of the concentration profiles of reactants $X$ (red) and $Y$ (black) and product $Z$ (blue). The top plot shows the initial configuration of a rectangular slug of $\mathrm{X}$ in a stream of $\mathrm{Y}$, centred at channel position $=0$. The left-hand plots correspond to $v_{\text {eof }}=0.05$ mm $\mathrm{s}-1$ with $v_{\mathrm{ph}}$ of all species $=0$ (diffusive mixing only). The right-hand plots correspond to $v_{\mathrm{eof}}=0.05 \mathrm{~mm} \mathrm{~s}^{-1}$ and $v_{\mathrm{ph}}(\mathrm{X})=0, v_{\mathrm{ph}}(\mathrm{Y})=0.76 \mathrm{~mm} \mathrm{~s}$ and $v_{\mathrm{ph}}(\mathrm{Z})=0.23 \mathrm{~mm} \mathrm{~s}$. In this case, mixing of $\mathrm{X}$ and $\mathrm{Y}$ occurs mainly as a result of the different electrophoretic velocities.

'flow-inject-flow' sequence described earlier, a slug of one reagent is produced within a flowing stream of a second reagent. We illustrate the main features of this mode by considering the behaviour of a slug of a reagent $X$ in a flowing stream of a reagent $\mathrm{Y}$ where $\mathrm{X}$ and $\mathrm{Y}$ can chemically react to give product $\mathrm{Z}$. We consider the situation where the reactant concentrations and the reaction rate constant are such that chemical reaction occurs fast, i.e. virtually immediately the reagents are locally mixed in a particular channel section. Using the simulation procedure described in Ref. 36, we are able to calculate the concentrations of all the species $\mathrm{X}, \mathrm{Y}$ and $\mathrm{Z}$ as a function of the distance $\mathrm{x}$ along the flow channel length for different times after the production of the initial rectangular slug of $\mathrm{X}$ in the stream of $\mathrm{Y}$. Input variables include the initial concentrations of $\mathrm{X}, \mathrm{Y}$ and $\mathrm{Z}$, the forward and reverse rate constants for the reaction $\mathrm{X}+\mathrm{Y}=\mathrm{Z}$, the EOF velocity and the electrophoretic velocities of $\mathrm{X}, \mathrm{Y}$ and $\mathrm{Z}$. The first simulation (Fig. 13) shows a series of time snapshots for the situation in which the electrophoretic velocities of $\mathrm{X}, \mathrm{Y}$ and $\mathrm{Z}$ are all zero (corresponding to these species all being uncharged). In this case, all species move with velocities equal to $v_{\text {eof }}$ and mixing (and hence reaction) occurs only by inter-diffusion at the leading and trailing edges of the slug of $\mathrm{X}$. For the wide slug and the short time shown in Fig. 11, the inter-diffusion and therefore the extent of the reaction, is low. The time for diffusional mixing across the slug (approximately $5 w_{\mathrm{s}}^{2} / D$, where $w_{\mathrm{s}}$ is the slug width) can be made shorter by reducing the slug width. In order to maximise the conversion to products, a series of many narrow slugs is therefore more effective than a single, broad slug. Elegant methods to achieve very narrow slug widths have been described. ${ }^{44}$

In the second simulation shown in Fig. 13, $v_{\mathrm{ph}}$ for $\mathrm{X}$ is set to zero, but $v_{\mathrm{ph}}$ for $\mathrm{Y}$ and $\mathrm{Z}$ are set to positive values corresponding to these species bearing positive charges. $Y$ now moves slowly with a velocity $v_{\text {eof }}$ whereas $\mathrm{X}$ moves faster with a velocity $v_{\text {eof }}+v_{\mathrm{ph}}$. In this situation, the slug of $\mathrm{X}$ becomes displaced relative to the concentration 'gap' in $\mathrm{Y}$ and mixing of $\mathrm{X}$ and $\mathrm{Y}$ therefore occurs relatively rapidly as a result of the different electrophoretic mobilities of the two species and results in reaction at the trailing edge of the slug of X. It should be noted that, in contrast to the diffusive mixing mode discussed above, mixing by differential electrophoretic mobilities does not result in dilution of the localised reagent concentrations. Fig. 14 shows the experimental realisation of this type of mixing and reaction. A slug of the uncharged ligand, pyridine-azo-dimethylaniline (PADA), is injected into a flowing stream of $\mathrm{Ni}^{2+}$ ions. As seen in the measured concentration profiles along the channel length, fast complexation to form the NiPADA ${ }^{2+}$ 

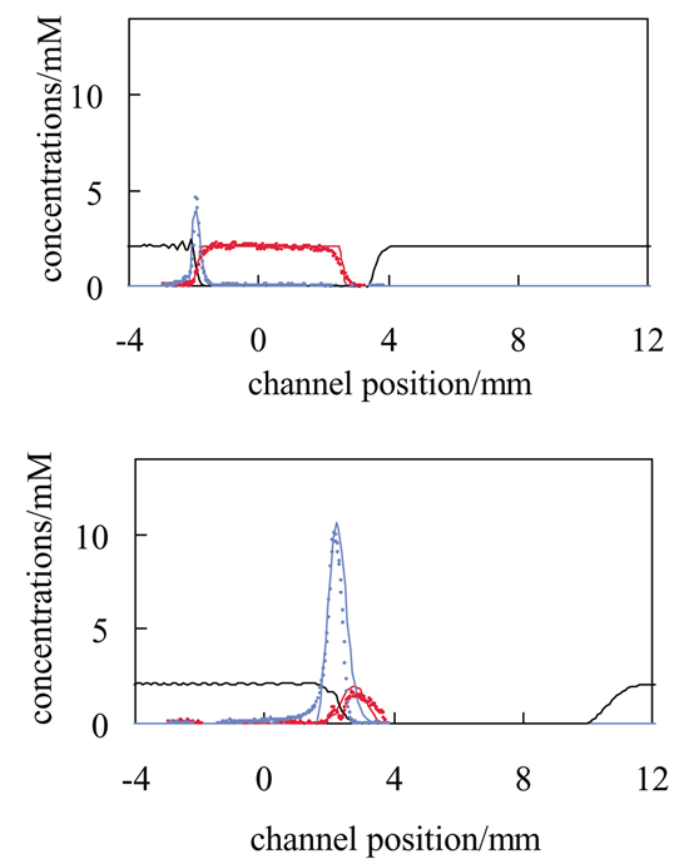

Figure 14. Comparison of simulated (solid lines) and measured (data points) concentrating profiles for the reaction between $\mathrm{Ni}^{2+}$ ions (black) and a slug of the ligand PADA (red) to form the $\mathrm{NiPADA}^{2+}$ complex (blue) at the tailing edge of the PADA slug. Concentration profiles of the coloured PADA and complex species were determined by analysis of digitised video images. The concentration profiles correspond to 10 (upper plot) and $70 \mathrm{~s}$ (lower plot) after PADA slug injection.

complex occurs at the trailing edge of the PADA slug and can be successfully modelled using the kinetic rate parameters measured for the forward (complexation) and reverse (complex dissociation) reactions. ${ }^{51}$

Following the time sequence shown in Fig. 14, it is possible to reverse the flow which moves the NiPADA ${ }^{2+}$ complex product peak back into the concentration gap of $\mathrm{Ni}^{2+}$. Since the complex formation reaction is reversible, the complex dissociates via the back reaction into PADA and $\mathrm{Ni}^{2+}$. This example, using a mechanistically very simple reaction, demonstrates the type of spatial and temporal control of chemical reactions that is achievable in micro reactors, but not in bulk solution reactors where all concentrations are uniform. The potential for detailed control of complex, multistep reactions is only just beginning to be explored. As discussed below, the empirical results for a wide range of reactions performed in micro reactors indicate that voltage control can alter the product yields and selectivity for many reactions. The full elucidation and optimisation of these effects will require detailed analysis using the principles described above. In addition to applications involving the control of synthetic reactions, micro reactors also have potential as 'chemical information chips'. Comparison of experimental concentration profile data for reactions for which diffusion coefficients, electrophoretic mobilities and rate constants are unknown with simulations such as those shown in Figs. 13 and 14 should enable the determination of the relevant physicochemical properties. Micro reactors therefore potentially offer a significant new tool in the armouries of both synthetic and physical chemists.

\section{Reactions performed in micro reactors}

\subsection{Liquid phase reactions}

Salimi-Moosavi et al. ${ }^{52}$ have demonstrated the synthesis of diazo dyes within a micro reactor. The authors have reacted 4-nitrobenzenediazonium tetrafluoroborate 1 with $\mathrm{N}, \mathrm{N}$ dimethylaniline $\mathbf{2}$ in a micro reactor fabricated from glass, to give the red diazo compound $\mathbf{3}$ (Scheme 1). The reagents were mobilised in the reactor using EOF in either a protic (methanol) or an aprotic (acetonitrile) solvent, to give conversions of 37 and $22 \%$, respectively.

Nitration reactions in organic synthesis are problematic because of the use of excess quantities of concentrated nitric and sulphuric acids. The reactions are extremely exothermic and it is hence difficult to control the temperature of such reactions when performed on a large scale. As a result, micro reactors have a considerable attraction for these reactions because the reactor enables excellent temperature control of the reaction.

Doku et al. ${ }^{53}$ have reported the nitration of benzene 4 in a<smiles>COc1ccccc1</smiles><smiles>Cc1ccc(/N=N/c2ccc([N+](=O)[O-])cc2)cc1</smiles>

Scheme 1.

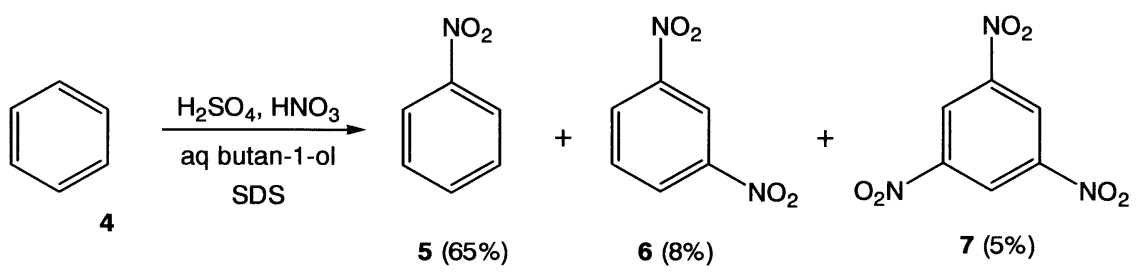




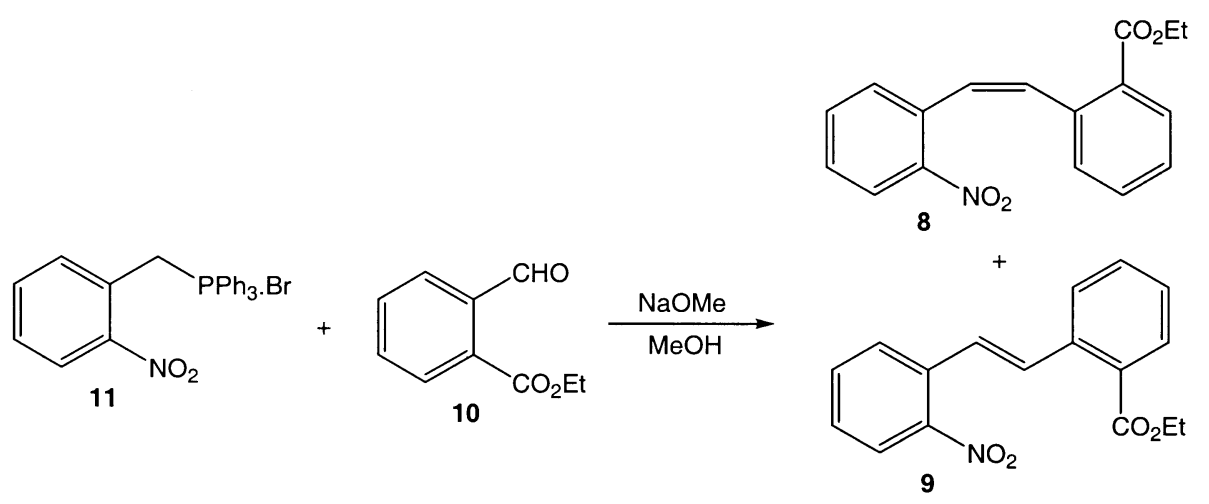

Scheme 3.

borosilicate glass micro reactor. The benzene was mobilised by electroosmotic flow as a microemulsion using the surfactant, sodium dodecyl sulphate (SDS). The nitronium ions, which were produced in situ by mixing sulphuric and nitric acids, underwent electrophoretic-induced mobility. A co-solvent, butan-1-ol, was used to enhance the solubility of the benzene in the aqueous system. The authors report that mononitration occurs in $65 \%$ conversion to give nitrobenzene 5 (Scheme 2) and that approximately $8 \%$ of 1,3dinitrobenzene $\mathbf{6}$ and $5 \%$ of 1,3,5-trinitrobenzene 7 were obtained. Importantly, Doku demonstrated that it is possible to mobilise a non-polar liquid, such as benzene, using EOF by dissolving it in a microemulsion.

Burns and Ramshaw ${ }^{54}$ have also investigated the nitration of benzene and toluene in a micro reactor. They have reported that the conversion has a linear relationship with temperature. More interestingly, they have demonstrated that the conversion can be considerably increased by reducing the dimensions of the micro reactor channels. They found that halving the capillary diameter from 250 to $130 \mathrm{~mm}$ more than doubled the rate of nitration. The flow rates were additionally determined to be critical, with faster flow rates giving higher conversions. The authors postulate that the increased flows promoted increased mixing within the channels.

Skelton and co-workers have reported the application of micro reactors, prepared from borosilicate glass, for the Wittig reaction. ${ }^{55,56}$ The authors used the micro reactor to prepare the cis- and trans-nitrostilbene esters 8 and 9 using the Wittig reaction (Scheme 3). A number of features such as stoichiometry and stereochemistry were investigated. When 2 equiv. of the aldehyde $\mathbf{1 0}$ to the phosphonium salt 11 were used in the reaction, a conversion of $70 \%$ was achieved. The micro reactor demonstrated an increase in reaction efficiency of $10 \%$ over the traditional batch synthesis. The reaction stoichiometry was subsequently reduced to $1: 1$, but using a continuous flow of reagents, as above, the conversion was poor (39\%). The conversion was increased to $59 \%$ using an injection technique, where slugs of the phosphonium salt $\mathbf{1 1}$ were injected into a continuous flow of the aldehyde $\mathbf{1 0 .}$

The research was further extended to investigate the stereochemistry of the reaction. The ratio of isomers 8 and 9 was controlled by altering the applied voltages to the reagent reservoirs within the device. The variation in the external voltage subsequently altered the relative reagent concentration within the device, producing $Z / E$ ratios in the region 0.57-5.21. In comparison, the authors report that, when a traditional batch synthesis was performed based on the same reaction time, concentration, solvent and stoichiometry, a $Z / E$ ratio of approximately $3: 1$ was observed. This demonstrated that significant control was possible in a micro reactor compared with batch reactions.

Sands and co-workers ${ }^{57}$ have recently reported the preparation of enamines in a micro reactor. Enamines are traditionally prepared under Dean and Stark conditions, where the ketone and secondary amine are heated to reflux in toluene. These conditions remove the water from the reaction to produce the equilibrium-dependent enamine. Using the micro reactor, cyclohexanone $\mathbf{1 2}$ was reacted with pyrrolidine 13 using methanol as the solvent, in the presence of dicyclohexylcarbodiimide (DCC), to form the enamine 14 in $42 \%$ conversion at room temperature (Scheme 4$)$. The authors postulate that the DCC reacts with the water that is produced in the reaction.
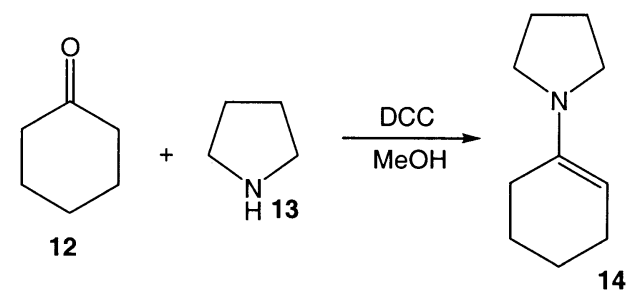

Scheme 4.

Wiles et al. ${ }^{58}$ have recently demonstrated the use of silyl enol ethers in the aldol reaction within a micro reactor. Quantitative conversion of the silyl enol ethers to $\beta$-hydroxyketones was observed in 20 min compared to traditional batch systems, where quantitative yields were only obtained when extended reaction times of up to $24 \mathrm{~h}$ were employed. One example involved the treatment of the TMS enol ether 15 with tetra- $n$-butylammonium fluoride (TBAF) to generate the tetra- $n$-butylammonium enolate $\mathbf{1 6}$ in situ, followed by condensation with $p$-bromobenzaldehyde $\mathbf{1 7}$ to give the $\beta$-hydroxyketone $\mathbf{1 8}$ in $100 \%$ conversion (Scheme 5). 


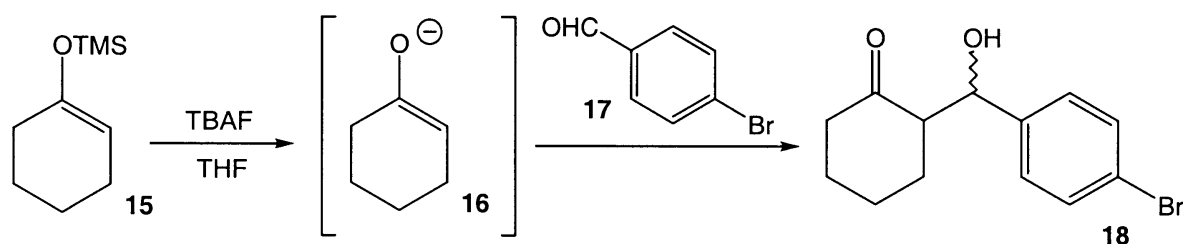

Scheme 5.<smiles>CC(C)=O</smiles>

Scheme 6.

Wiles et al. ${ }^{59}$ have reported the preparation of the enolates from a series of 1,3-diketones using an organic base, and their subsequent reaction with a variety of Michael acceptors such as $\mathbf{1 9}$ to afford 1,4-addition products within a micro reactor (Scheme 6).

When using a continuous flow of the reagents $\mathbf{1 9}$ and $\mathbf{2 0}$, $15 \%$ conversion to the adduct 22 was observed, compared with $56 \%$ when the diketone 21 was reacted with 19 forming the Michael adduct 23. The authors, however, demonstrated enhancements in conversions through the application of the stopped flow technique. This procedure involved the mobilisation of reagents through the device for a designated period of time, using an applied field, and the flow was subsequently paused by the removal of the applied field, prior to re-applying the field. Using the regime of $2.5 \mathrm{~s}$ on and $5 \mathrm{~s}$ off, the conversion to the product $\mathbf{2 2}$ was improved to $34 \%$, while lengthening the stopped flow period to $10 \mathrm{~s}$, resulted in a further increase to $100 \%$. This was compared to the preparation of 23 , in which the regime of $2.5 \mathrm{~s}$ on and $5 \mathrm{~s}$ off resulted in an increase in conversion to $95 \%$. This demonstrated that the enolate of 2,4-pentanedione 21 was more reactive than the corresponding enolate of benzoyl acetone 20. The authors propose that the observed increase in conversion, when using the technique of stopped flow, was due to an effective increase in residence time within the device and hence an increase in the diffusive mixing of the reagent streams.

Jenson et al. ${ }^{60}$ have reported a photochemical reaction within a micro reactor. The reactor was fabricated by bonding a patterned silicon wafer to a quartz wafer, the advantage of this fabrication technique being that the quartz substrate allows reaction and detection using UV light of lower wavelengths than permitted by Pyrex substrates.

The authors investigated the pinacol formation reaction of benzophenone $\mathbf{2 4}$ in propan-2-ol (Scheme 7). The reaction is known to follow a radical reaction pathway, ${ }^{61}$ and it is reported that the longer the residence time of the reaction, the greater the conversion to benzopinacol 25. The authors report that there was no detectable product formation for flow rates $>10 \mu \mathrm{min}^{-1}$. With reduced flow rates, having larger residence times, the conversion improves because the<smiles>CC(=O)CCCCCCCCCCC(C)O</smiles>

Scheme 7.<smiles>Cc1cc(O)cc(O)c1</smiles><smiles>O=[N+]([O-])c1ccc([N+](=O)[O-])cc1</smiles>

26<smiles></smiles>

Scheme 8. 
<smiles>O=C(O)CCNC(F)F</smiles>

28<smiles>NCCC(=O)OCC(=O)O</smiles>

DCC, DMF

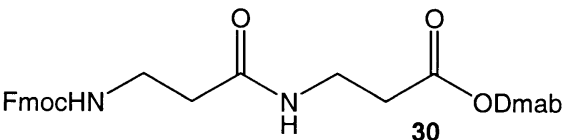

Scheme 9.

amount of light absorbed increases, and there is therefore sufficient time for the excited species to diffuse and react with the benzophenone. The authors report conversions of up to $60 \%$ when using flow rates of $4 \mu 1 \mathrm{~min}^{-1}$.

Hisamoto and co-workers ${ }^{62}$ have described the first example of a phase transfer reaction in a micro reactor. These authors have successfully conducted a phase transfer diazo coupling reaction in which a solution of 5-methylresorcinol $\mathbf{2 6}$ in ethyl acetate was reacted with an aqueous solution of 4-nitrobenzenediazonium tetrafluoroborate $\mathbf{1}$ to form the azo dye 27 (Scheme 8). Syringe pumps were used to move the reagents around the reactor manifold and the authors report that the product was isolated in $100 \%$ yield.

Watts et al. have recently demonstrated the first example of a multistep synthesis in a micro reactor where they have used their micro reactor devices in peptide synthesis. ${ }^{63}$ The authors evaluated their device using a carbodiimide coupling reaction of Fmoc- $\beta$-alanine 28 with the amine 29 to give the dipeptide 30 (Scheme 9). When stoichiometric quantities of the reagents were used, only ca $10 \%$ conversion to dipeptide the $\mathbf{3 0}$ was achieved. By using two equiv of DCC, however, an increase in conversion to ca $20 \%$ was observed and by applying a stopped flow technique $(2.5 \mathrm{~s}$ injection length with stopped flow for $10 \mathrm{~s}$ ), the conversion of the reaction was further increased to approximately $50 \%$. Using five equiv of DCC, a conversion of up to $93 \%$ of the dipeptide $\mathbf{3 0}$ was obtained using the stopped flow technique.
The authors also demonstrated that the dipeptide could be prepared from pre-activated carboxylic acids. They report that the reaction of the pentafluorophenyl (PFP) ester of Fmoc- $\beta$-alanine $\mathbf{3 1}$ with the amine $\mathbf{2 9}$ gave the dipeptide 30 quantitatively in $20 \mathrm{~min}$ (Scheme 10). This represented a significant increase in yield compared with the traditional batch synthesis, where only a $50 \%$ yield was obtained in $24 \mathrm{~h}$.

Having demonstrated that peptide bonds could be successfully formed when using a micro reactor, the authors then found that they could extend the methodology to the preparation of longer-chain peptides. Using the micro reactor, the Dmab ester of Fmoc- $\beta$-alanine 32 was reacted with one equiv of piperidine or 1,8-diazabicyclo(5.4.0)undec-7-ene (DBU) to give the free amine 29 in quantitative conversion. This is in comparison to the solid phase peptide synthesis where $20 \%$ piperidine in DMF is frequently employed. ${ }^{64,65}$ The authors then reacted the amine in situ with the pentafluorophenyl ester $\mathbf{3 3}$ to give the dipeptide $\mathbf{3 4}$ (Scheme 11) in $96 \%$ overall conversion.

Having shown that more complex peptides could be produced by removal of the $\mathrm{N}$-protecting group, the authors then demonstrated that they could remove the Dmab ester using hydrazine. The reaction of the Dmab ester 32 with 1 equiv. of hydrazine gave quantitative deprotection to afford the carboxylic acid $\mathbf{2 8}$ (Scheme 12). This is in comparison to the solid phase peptide synthesis where $2 \%$ hydrazine in DMF is generally used to effect deprotection. ${ }^{66}$

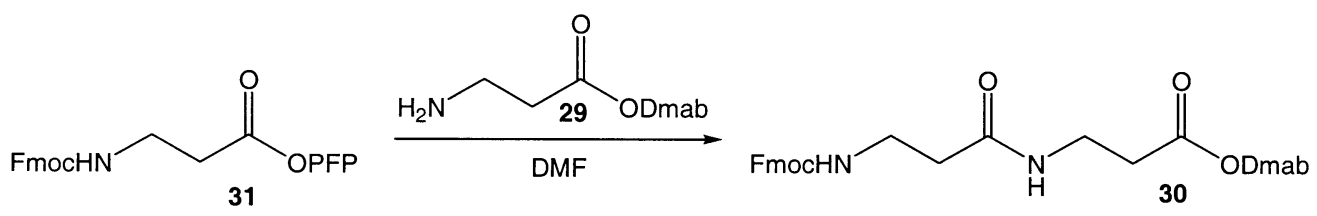

Scheme 10.

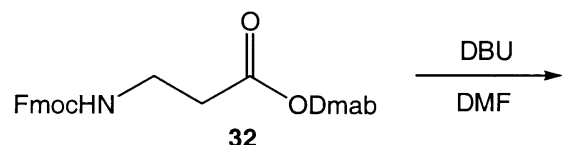

32<smiles>NCCC(=O)O[18O]</smiles>

29

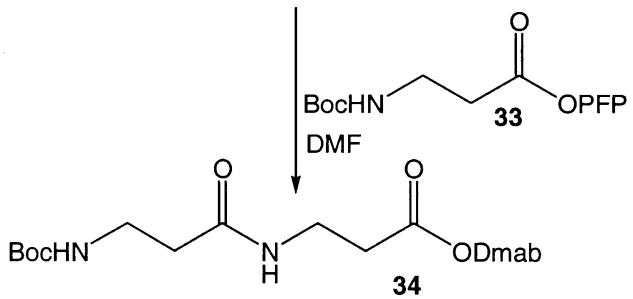




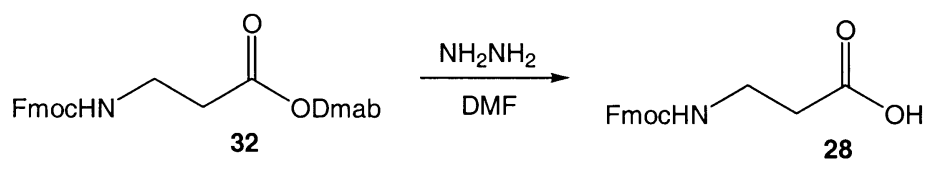

Scheme 12.

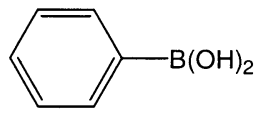

37

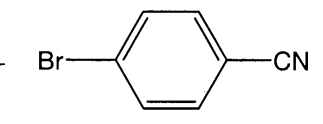

36

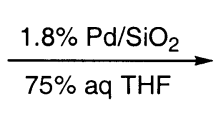

$75 \%$ aq THF

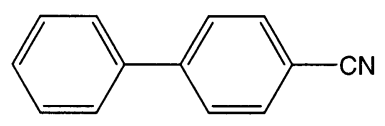

35

Scheme 13.

\subsection{Catalytic reactions}

Greenway et al. have demonstrated the Suzuki reaction within a micro reactor device. ${ }^{67}$ This represented an example of heterogeneous catalysis where $1.8 \%$ palladium on silica was placed in the central channel of the micro reactor. The catalyst was immobilised between microporous silica frits prepared from potassium silicate and formamide. The micro reactor was optimised using flow injection analysis principles, producing a conversion of $67 \%$ of the cyanobiphenyl 35 product at room temperature. The flow injection method adopted allowed the periodic injection of the aryl halide 36 into a continuous flow of the phenylboronic acid 37 (Scheme 13). Traditionally, tetrahydrofuran (THF) is used as the solvent in this reaction. As has been found with many organic solvents, however, THF has very low natural EOF properties and for this reason, it was mixed with water $(75: 25)$ for use in the experiment. The yields obtained were comparable with Suzuki reactions on a batch scale using homogeneous catalysis. Importantly, there were negligible levels of the palladium catalyst in the product, which was demonstrated using inductively coupled-mass spectrometry (ICP-MS), this illustrating that the catalyst was not leaching from the reactor.

One of the interesting observations of the reaction was that, unlike conventional Suzuki reactions, an additional base was not required. Although the exact reason for this is not clear, it is postulated that the electric field may be sufficient to cause ionisation of the water at the metal surface. It is feasible that the hydroxide formed in this way may be sufficient to perform the function of the conventional organic or inorganic base. Alternatively, it has been subsequently proposed that a more basic environment may be formed at the surface of the micro reactor.

Wilson and McCreedy ${ }^{68}$ have reported the use of a micro reactor to perform the dehydration of hexan-1-ol to hex-1ene, using a sulphated zirconia catalyst. The micro reactor was fabricated from a glass plate, which was etched using photolithography. A PDMS top block, with pre-drilled holes to act as reservoirs for the reagents, was then aligned with the channel geometry. In order to introduce the catalyst into the micro reactor, it was dusted over the surface of the PDMS face before the base plate was joined to the top plate. This process immobilised the catalyst, while simultaneously increasing its surface area. The overall effect was to produce a catalytically-active wall of the microchannel. A heater, fabricated from Nichrome wire, was also immobilised in the top plate. Pumping was produced with a syringe pump and the products were analysed by gas chromatography (GC). The conversion of hexan-1-ol $\mathbf{3 8}$ to hex-1-ene 39 was between 85 and $95 \%$ and no additional products were detected (Scheme 14). This yield is extremely good when compared to the $30 \%$ yield expected for the industrially used process.

The reaction was also applied to ethanol. At a reaction temperature of $155^{\circ} \mathrm{C}$ and using a syringe pump at a flow rate of $3 \mu \mathrm{min}^{-1}$, the product collected was found to contain $68 \%$ ethene, $16 \%$ ethane and $15 \%$ methane, together with trace amounts of ethanol. When electroosmotic pumping was used, the flow rate obtained was between 0.9 and $1.1 \mu 1 \mathrm{~min}^{-1}$ at a field strength of $200 \mathrm{~V} \mathrm{~cm}^{-1}$. The only detectable product was methane, indicating that the reaction had progressed beyond dehydration to complete cracking of the ethanol. Additionally, trace amounts of methanol were present in the product. It is proposed that the slow flow rate of the electroosmotic pumping method results in longer residence times in the reactor, this offering a significant advantage over the syringe pump. EOF however cannot be applied to all reactions because organic reactants, such as hexanol, exhibit no natural EOF under an applied potential.

O'Sullivan et al. ${ }^{69}$ have recently investigated the KumadaCorriu reaction in a pressure-driven micro reactor. The reactor was constructed by placing a plug of catalyst into a length of polypropylene tubing. The catalyst was held in place using plugs of glass wool. Standard HPLC connectors allowed one end of the tubing to be connected to a syringe. A syringe pump was used to drive a pre-mixed solution containing equimolar quantities of the aryl halide and Grignard reagent through the reactor.

The authors reacted $p$-bromoanisole $\mathbf{4 0}$ with phenylmagnesium bromide 41, in the presence of the nickel catalyst $\mathbf{4 2}$,
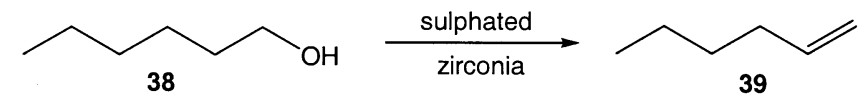


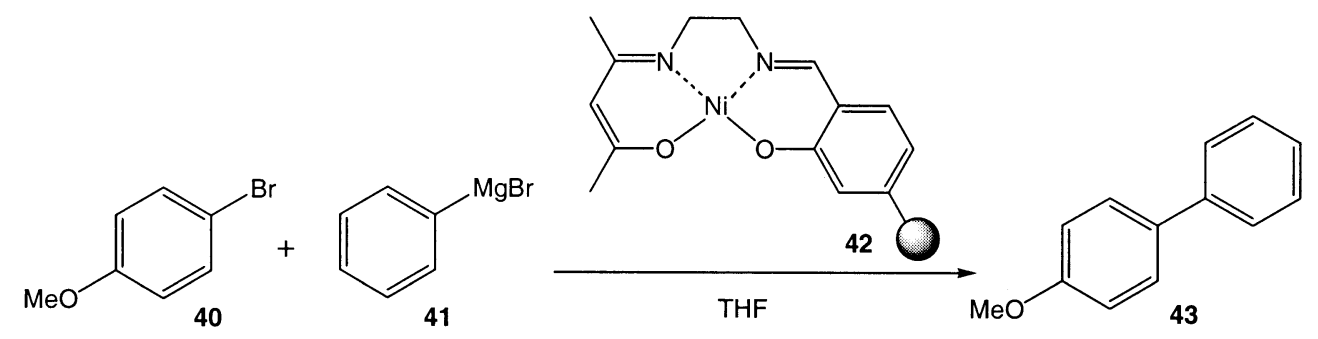

Scheme 15.

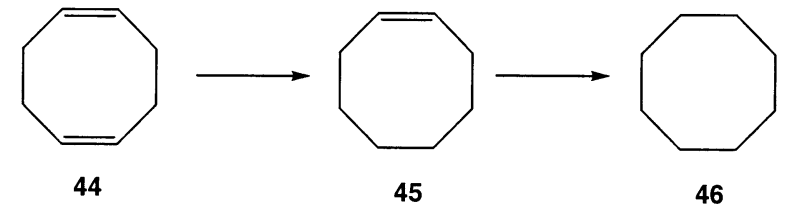

Scheme 16.

which was supported on Merrifield resin, to give 4-methoxybiphenyl 43 (Scheme 15). When the reaction was conducted in the micro reactor, an enhanced reaction rate was observed compared to the batch reactions. Since the concentrations were the same in the both cases, the authors postulate that the dimensions of the micro reactor are solely responsible for the enhanced rate of reaction.

\subsection{Gas phase reactions}

Hönicke and co-workers ${ }^{70}$ have reported the gas phase partial oxidation of cyclic dienes, to their corresponding monoalkenes, over palladium and ruthenium/zinc catalysts. The micro reactors consisted of aluminium wafers, with mechanically-etched channels, which were activated by anodic oxidation to obtain a porous oxide layer, which was used as the catalyst support. Impregnation of an organic solution of palladium(II) acetylacetonate resulted in microchannels consisting of an $18 \mu \mathrm{m}$ thick layer of $0.18 \% \mathrm{Pd}$ catalyst. The wafers were then stacked in a stainless steel housing to form a micro reactor consisting of 672 microchannels for a stream of reagents to pass through. The authors used the device to investigate the hydrogenation of 1,5-cyclooctadiene 44 to cyclooctene 45 (Scheme 16). The diene 44 was vapourised and mixed with hydrogen, before being passed through the micro reactor at a temperature of $150^{\circ} \mathrm{C}$. By increasing the residence time of the reaction from 35 to $115 \mathrm{~ms}$ the authors report that the conversion increased from 75 to $99.5 \%$. Although the increased residence time resulted in increased quantities of cyclooctane $\mathbf{4 6}$ being formed, the selectivity of cyclooctene $\mathbf{4 5}$ decreased from 99.5 to $98 \%$ under these conditions.

The authors used the same device to investigate the hydrogenation of $c, t, t, 1,5,9$-cyclododecatriene 47 to the cyclododecenes 48 and 49 (Scheme 17). At a temperature of $150^{\circ} \mathrm{C}$, a selectivity of 85 to $90 \%$ was reported, where the conversion was approximately $90 \%$. The selectivity of this reaction was lower than the previous example because of the formation of the by-products 50-52. It was demonstrated, however, that there was a selectivity advantage of the micro reactor compared to a fixed-bed reactor.

The catalytic hydrogenation of benzene 4 was also investigated (Scheme 18), but complete reduction to cyclohexane 53 was observed to take place when using the Pd catalyst. The authors report that hydrogenation of benzene to cyclohexene $\mathbf{5 4}$ was accomplished using a micro reactor system consisting of a ruthenium/zinc catalyst, which was incorporated into the micro reactor using the same methodology, but the conversions were reported to be low (ca 10\%), with a maximum selectivity of $36 \%$.

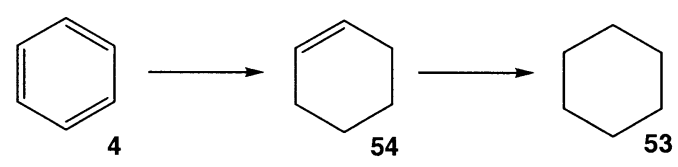

Scheme 18.

The use of elemental fluorine in organic synthesis is problematic as a result of the difficulties associated with the safe handling of gaseous fluorine. ${ }^{71,72}$ In addition, fluorination reactions are generally extremely exothermic and it is difficult to control the temperature of such reactions when performed on a large scale. Micro reactors have a considerable attraction for direct fluorination processes because there is only a small amount of fluorine in the reactor at any given time. The micro reactor enables

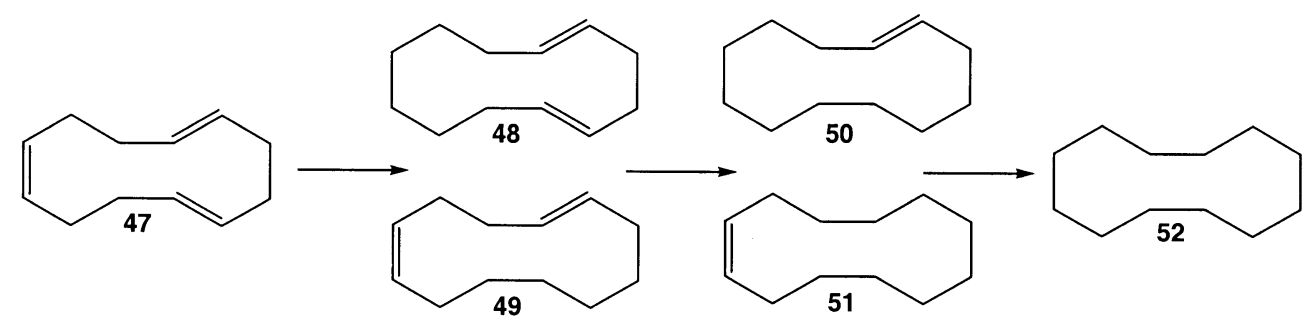


<smiles>CCOC(=O)CC(C)=NC(=O)C(F)C(=O)OCC</smiles><smiles>CCOC(=O)C(Cl)C(=O)C(F)(Cl)C(C)=O</smiles>

Scheme 19.

excellent temperature control of the reaction as well as an opportunity for scale up, by the simultaneous use of many such reactors.

Chambers and Spink ${ }^{73,74}$ have reported the use of micro reactors for the fluorination and perfluorination of organic compounds using elemental fluorine. A nickel or copper micro reactor was used for the investigation and the liquid reactants and solvents were introduced into the reaction chamber via a syringe using a syringe-pump. Fluorine, in a nitrogen carrier gas, was introduced from a cylinder using a mass-flow controller. The liquid-gas mixing proceeded via 'cylindrical flow', where the liquid forms an outer cylinder coating the reactor surface with the gas flowing through the centre. This flow regime has enormous benefits in that it provides very large surface-to-volume ratios for the liquid phase, producing a very efficient reaction over a short distance. The products were trapped in a tube, which was cooled with either a salt/ice bath $\left(0^{\circ} \mathrm{C}\right)$ or an acetone/carbon dioxide bath $\left(-78^{\circ} \mathrm{C}\right)$. The fluorination of $\beta$-dicarbonyl compounds proceeded with a high efficiency using $10 \%$ fluorine in nitrogen at $5^{\circ} \mathrm{C}$ and with formic acid as the solvent. Ethyl acetoacetate $\mathbf{5 5}$ was fluorinated in $99 \%$ conversion to give ethyl 2-fluoroacetoacetate 56 while ethyl 2-chloroacetoacetate $\mathbf{5 7}$ was fluorinated in $90 \%$ conversion, yielding ethyl 2-chloro-2-fluoroacetoacetate 58 (Scheme 19). Importantly, under these conditions, no perfluorination of the substrates was observed, with only the monofluorinated derivatives being isolated. The authors report that the bulk fluorination of ethyl 2-chloroacetoacetate $\mathbf{5 7}$ gives only a low conversion to $\mathbf{5 8},{ }^{75}$ illustrating that the flow system is more efficient. This illustrates the catalytic effect of the fluorinated metal surface.

The sulphur pentafluoride derivative $\mathbf{5 9}$ was prepared in $75 \%$ yield by the reaction of the disulphide $\mathbf{6 0}$ with $10 \%$ fluorine in nitrogen, using acetonitrile as the solvent (Scheme 20). Similarly, treatment of the trifluoride 61 with excess fluorine gave the sulphur pentafluoride derivative 62 in $44 \%$ yield.

Perfluorination reactions were found to require an additional heating stage for the reaction to go to completion. The reaction of the tetrahydrofuran derivative $\mathbf{6 3}$ with $50 \%$ fluorine in nitrogen at $280^{\circ} \mathrm{C}$ gave the perfluorinated compound 64 in $91 \%$ yield (Scheme 21 ). In conventional reactions, cobalt trifluoride would be used to perfluorinate hydrocarbons. ${ }^{76}$ Some of the reactions carried out by the authors, however, required much lower temperatures than would be expected if this compound was used.

Jenson has demonstrated the direct fluorination of aromatic compounds in a micro reactor, a process difficult to perform on a conventional scale. ${ }^{77}$ The reactor was fabricated from silicon and capped with Pyrex using anodic bonding. The surfaces of the reactor, which were in contact with the reagents, were coated with a nickel film using a metal deposition technique. The authors have used the micro reactor in the fluorination of toluene $\mathbf{6 5}$ at room temperature (Scheme 22). Using 10 equiv. of fluorine, in methanol as the solvent, the authors report an $80 \%$ conversion to give<smiles>O=[N+]([O-])c1cccc(SSc2cccc([N+](=O)[O-])c2)c1</smiles><smiles>CC(C)[Pb]</smiles><smiles>O=[N+]([O-])c1cccc(S(F)(F)(F)(F)F)c1</smiles>

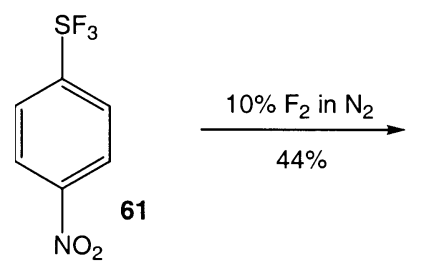<smiles>O=[N+]([O-])c1ccc(S(F)(F)(F)(F)F)cc1</smiles>

Scheme 20.

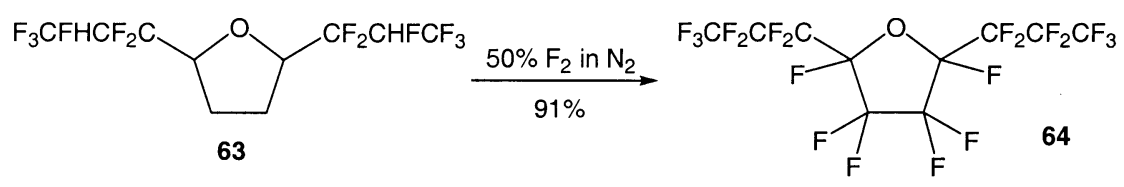




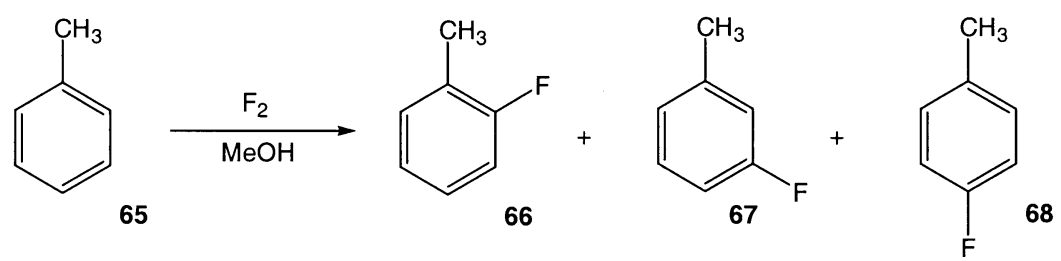

Scheme 22.

the monofluorinated toluenes. The substitution pattern of the ortho-66, meta-67 and para-68 isomers was determined to be $4: 1: 2$ by GC.

Srinivasan et al. $^{78}$ performed the partial oxidation of ammonia using a silicon-based micro reactor. Integrated heaters as well as flow and temperature sensors were fabricated into the sub-mm flow channels. The platinum catalyst was deposited in the reaction channel by electron-beam evaporation via a shadow mask. The gaseous reactants were fed from cylinders into the micro reactor by external mass-flow controllers, which maintained the desired flow rates. The product composition was continuously monitored using a mass spectrometer. The authors reported a change in the micro reactor exhaust composition over a range of temperatures and flow rates and they also demonstrated that the conversion and selectivity behaviour of conventional reactors could be reproduced in a micro reactor.

The effective heat transfer of micro reactors provides very accurate temperature control for both exothermic and endothermic reactions, thus eliminating undesired side reactions. An example has been reported by Hessel et al., ${ }^{79}$ who demonstrated that a micro reactor could be used to prepare hydrogen cyanide via the Andrussow route. In traditional laboratory reactions, the hydrogen cyanide is reported to hydrolyse to ammonia. The use of a microheat exchanger in this experiment, however, prevented this further reaction occurring.
As a final summary to this section, we list in Table 2 the reactions that are currently being investigated in micro reactors.

\section{Potential applications of micro reactors}

This review has summarised a small yet significant area of scientific research that is poised to challenge our understanding and future applications of reaction chemistry. To date, the outcome of the reported research has confirmed that micro reactor methodology is applicable to performing both gas and liquid phase reaction chemistry. But the question which remains are: is there more beyond just doing smaller than what can already be performed by traditional methodology? From the work cited in this article, the evidence is that the unique modus operendi of micro reactors, namely the low-volume spatial and temporal control of reactants and products in a laminar flow diffusive mixing environment in which distinctive thermal and concentration gradients exist, offers a novel method for the chemical manipulation and generation of products. In short, micro reactors are new tools with which to generate molecules and increase our knowledge of complex chemical processes.

The technology is still in its early development stage and it would be presumptuous at this point to expand too far on the potential applications that micro reactors will find, but some early trends are clear. In the authors' experience, reactions

Table 2. Reactions conducted in a micro reactor

\begin{tabular}{|c|c|c|c|c|c|}
\hline Reaction & Chip material & Solvent & Conversion $(\%)$ & Comments & Reference \\
\hline Suzuki & Glass & aq THF & 67 & EOF & 67 \\
\hline Kumada coupling & Polypropylene & THF & 60 & Syringe pump & 69 \\
\hline Aldol & Glass & $\mathrm{THF}$ & 100 & EOF & 58 \\
\hline Nitration & Glass & Benzene & 65 & $\mathrm{EOF}$ & 53 \\
\hline Wittig & Glass & $\mathrm{MeOH}$ & $39-59$ & EOF & 55,56 \\
\hline Enamine & Glass & $\mathrm{MeOH}$ & 42 & EOF & 57 \\
\hline Ugi four component coupling & Glass & $\mathrm{MeOH}$ & & EOF & \\
\hline Peptide synthesis & Glass & $\mathrm{DMF}$ & 100 & EOF & 63 \\
\hline Synthesis of pyridazinones & Glass & $\mathrm{EtOH} / \mathrm{AcOH}$ & 30 & EOF & \\
\hline $\begin{array}{l}\text { Synthesis of amides from } \\
\text { amines and acid chlorides }\end{array}$ & Glass & DCM & 77 & EOF & \\
\hline Diazo coupling & Glass & $\mathrm{MeOH}, \mathrm{MeCN}$ & 37,22 & EOF & 52 \\
\hline Aminothiazole synthesis & Glass & NMP & $58-100$ & EOF & \\
\hline Knoevenagel & Glass & $\mathrm{MeOH} / \mathrm{H}_{2} \mathrm{O}$ & $59-68$ & EOF & \\
\hline Hantzsch thiazole synthesis & Glass & NMP & $58-100$ & EOF & \\
\hline Michael addition & Glass & $\mathrm{EtOH}$ & $95-100$ & EOF & 59 \\
\hline $\mathrm{S}_{\mathrm{N}} 2$ alkyl halide & Glass & $\mathrm{DMF} / \mathrm{H}_{2} \mathrm{O}$ & 25 & EOF & \\
\hline Dehydration & Glass/PDMS & $\mathrm{EtOH}$ & $85-95$ & EOF or syringe pump & 68 \\
\hline Photochemical & Silicon/quartz & $\left(\mathrm{CH}_{3}\right)_{2} \mathrm{CHOH}$ & 60 & Syringe pump & 60 \\
\hline Phase transfer & Glass & EtOAc & 100 & Syringe pump & 62 \\
\hline Fluorination & $\mathrm{Ni}$ or $\mathrm{Cu}$ & Nitrogen gas & $90-99$ & Syringe pump & 73,74 \\
\hline Fluorination & Silicon/pyrex & $\mathrm{MeOH}$ & 80 & Syringe pump & 77 \\
\hline
\end{tabular}




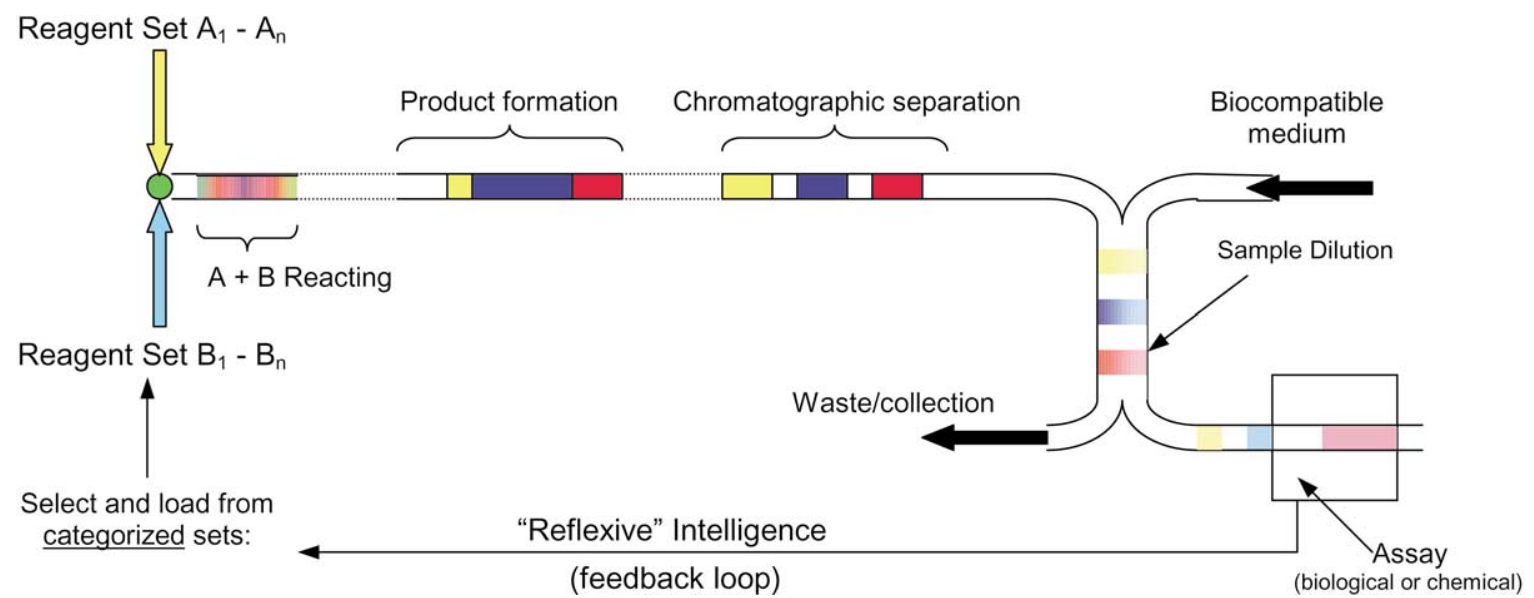

Figure 15. Integration of a micro reactor with a biological assay system.

performed in a micro reactor invariably generate relatively pure products in high yield, in comparison to the equivalent bulk reactions, in much shorter times and in sufficient quantities to perform full instrumental characterisation. One of the immediate and obvious applications is therefore in drug and process discovery, where the generation of compounds either with different reagents or under variable conditions is an essential factor. In addition, the opportunity to establish optimal chemical processes including reaction and formulation is an exciting capability of the technology, which could readily be married to appropriate analytical monitoring. An interesting twist to the chemistry carried out to date in the author's laboratories in not just the opportunity to separate reactants and products in real time but also the capability to manufacture and use reagents in situ. Perhaps more intriguing, is what new angles micro reactors bring to reaction chemistry and these are only now just emerging. Extending the heterogeneous catalyst work already described $^{64-66}$ one can see how immobilised or supported reagents could be placed within a device to impart functionality to a reaction whilst maintaining spatial and temporal control. This concept could be extended to consider selfassembly methods for generating surface-specific properties such as shape and energy or colloidal-based processes. Such concepts could also be easily extended to include biological functionality and integration. One intriguing aspect of electokinetically-operated micro reactors is anecdotal electrochemical effect that will undoubtedly yield novel chemistries in the next few years.

This review would not be complete without some estimate of the impact of this technology. The success of pharmaceutical companies resides largely on synthesis and screening of novel chemical entities representative of the universe of drug-like compounds, which may be of the order of $10^{200}$ compounds or about $10^{40}$ chemotypes, and to optimise selected leads to marketable drugs. Clearly, such a leadgeneration task is beyond terrestrial resources. In an industry where development costs are extraordinarily high and attrition rates from lead generation onwards are about $98 \%$, careful lead selection and ruthless pressure to shorten optimisation cycle times are, therefore, critical for survival. In addition to this diligence, new technology that would enable a cost-neutral upward step-change in the number of lead candidates (and thus choice of a better lead) and/or optimisation speed (to reduce time to market and extend patent life) would provide a distinct competitive advantage. The ability of the micro reactor to provide the opportunity for a range of controls over the chemistry environment, directly through device resident actuators, has already been described. A microchannel system also provides a potential separation column and a non-turbulent environment for partition between solvents. Integration of a micro reactor device, via purification and optional phase exchange elements, to one of the many highly sensitive microchannelbased biological assay systems may therefore not only be possible, but may also address some of the industries' potential requirements. Apart from the greatly reduced reaction times demonstrated for the micro reactors, handling times to assay and chemical reagent costs are virtually eliminated. In fact, with computer control and access to a range of categorised reagents, real-time prospecting to find and enhance leads may be possible. This paradigm is shown diagramatically in Fig. 15.

In lead optimisation using conventional batch technology, irrespective of whether parallel or iterative mode is chosen, validation and optimisation of reactions tends to be the ratelimiting step. Based on the model described in Fig. 15, however, it can be seen that, if the biological assay was replaced by a chemical assay, and the conditions not the reagents are varied, then an auto-optimisation could be carried out. Replication of the optimised channel as a parallel bundle could then provide a means of amplifying the amounts of material generated, even up to the quantities required for in vivo biology. In effect, chemistry is probably poised for the same sort of revolution that has taken place in electronics, biochemical screening and molecular biology, to name but a few examples. This is essentially a realisation that the desired product is usually information rather than a tangible, storable product, although here we can see that micro reactors can deliver both. This could enable the 'Moore's Law' environment for data generation required to support a cheminformatic approach to chemical lead discovery that is truly the counterpart of the bioinformatic discovery of biological targets from the human genome. It may also, at last, disconnect the chemist from the sheer slow drudgery of manual chemistry!

Clearly the mass of material generated in the type of micro 
reactor described is very low and with continuous use could produce up to $1 \mathrm{~g} /$ day. The engineering aspects of parallel scaling out, ie maintaining the advantages of a small reaction zone whilst achieving volume, have been elaborated. This aspect of volume production together with the concept of make and use (i.e. no inventory) and localised distribution rather than bulk manufacture, storage, shipping and delivery are important factors. Perhaps the more compelling driver to develop localised production facilities based on micro reactors is the economic benefits of efficient flexible production that is responsive to market demands.

\section{Concluding remarks}

Accept it or not, micro reactor chemistry exists and is currently showing great promise as a novel method on which to build new chemical technology and processes. One of the current barriers to testing the methodology would seem to be system availability and the authors are currently planning to make the technology more accessible to the wider community. From whichever camp, sceptic or enthusiast, in the scientific community, the real value of micro reactors needs to be established and readers wishing to express their views on this topic are invited to contact the authors of this review.

\section{Acknowledgements}

We wish to thank EPSRC, UK (X. Z.) and Novartis Pharmaceuticals (P. W.) for financial support.

\section{References}

1. Bradley, D. Eur. Chem. 1999, 1, 17.

2. Fletcher, P. D. I.; Haswell, S. J. Chem. Br. 1999, 35, 38.

3. Cowen, S. Chem. Ind. 1999, 584.

4. McCreedy, T. Chem. Ind. 1999, 588.

5. Barrow, D.; Cefai, J.; Taylor, S. Chem. Ind. 1999, 591.

6. Cooper, J.; Disley, D.; Cass, T. Chem. Ind. 2001, 653.

7. Haswell, S. J.; Middleton, R. J.; O’Sullivan, B.; Skelton, V.; Watts, P.; Styring, P. Chem. Commun. 2001, 5, 391.

8. IMRET 5: Proceedings of the Fifth International Conference on Microreaction Technology; Matlosz, M., Ehrfeld, W., Baselt, J. P., Eds.; Springer: Berlin, 2002.

9. Ehrfeld, W.; Hessel, V.; Löwe, H. Microreactors: New Technology for Modern Chemistry; Wiley-VCH: Weinheim, Germany, 2000.

10. Haswell, S. J. Analyst 1997, 112, 1R.

11. Manz, A.; Harrison, D. J.; Verpoorte, E.; Fettinger, J. C.; Ludi, H.; Widmer, H. M. Chimia 1991, 45, 103.

12. Manz, A.; Harrison, D. J.; Verpoorte, E.; Widmer, H. M. $A d v$. Chromatogr. 1993, 33, 1.

13. Manz, A.; Effenhauser, C. S.; Burggraf, N.; Verpoorte, E.; Raymond, D. E.; Widmer, H. M. Anal. Mag. 1994, 22, M25.

14. Proceedings of the Micro Total Analytical Systems '98 Workshop; Harrison, D. J., van den Berg, A., Eds.; Kluwer Academic: Dordrecht, 1998.

15. van den Berg, A.; Lammerink, T. S. J. Top. Curr. Chem. 1998, 194, 21.

16. Harrison, D. J.; Fluri, K.; Seiler, K.; Fan, Z. H.; Effenhauser, C. S.; Manz, A. Science 1993, 261, 895.
17. Jacobson, S. C.; Hergenroder, R.; Koutny, L. B.; Ramsey, J. M. Anal. Chem. 1994, 66, 1114.

18. Woolley, A. T.; Hadley, D.; Ladre, P.; DeMello, A. J.; Mathies, R. A.; Northrup, M. A. Anal. Chem. 1996, 68, 4081.

19. Woolley, A. T.; Mathies, R. A. Anal. Chem. 1995, 67, 3676.

20. Woolley, A. T.; Sensabaugh, G. F.; Mathies, R. A. Anal. Chem. 1997, 69, 2181.

21. Jacobsen, S. C.; Ramsey, J. M. Anal. Chem. 1996, 68, 720.

22. Effenhauser, C. S.; Paulus, A.; Manz, A.; Widmer, H. M. Anal. Chem. 1994, 66, 2949.

23. Schmalzing, D.; Adourian, A.; Koutny, L.; Ziagura, L.; Matsudaria, P.; Ehrlich, D. Anal. Chem. 1998, 70, 2303.

24. Spence, D. M.; Crouch, S. R. Anal. Chem. 1998, 358, 95.

25. Schoot, B. H.; Jeanneret, S.; Berg, A. Anal. Meth. Instrum. 1993, 1,38 .

26. Madou, M. Fundamentals of Microfabrication; CRC: Boca Raton, 1997.

27. McCreedy, T. TrAC 2000, 19, 396.

28. McCreedy, T. Anal. Chim. Acta 2001, 427, 39.

29. Katana 5040 High End Image Setter. For information see: www.screen.co.uk.

30. Broadwell, I.; Fletcher, P. D. I.; Haswell, S. J.; McCreedy, T.; Zhang, X. Lab on a Chip 2001, 1, 66.

31. Paul, P. H.; Garguilo, M. G.; Rakestraw, D. J. Anal. Chem. 1998, 70, 2459.

32. Overbeek, J. Th. G. Colloid Science; Kruyt, H. R., Ed.; Elsevier: Amsterdam, 1952; Vol. 1, p 195 Chapter V.

33. Rice, C. L.; Whitehead, R. J. Phys. Chem. 1965, 69, 4017.

34. Hunter, R. J. Zeta Potential in Colloid Science; Academic: London, 1981.

35. Jednacak, J.; Pravdic, V.; Haller, W. J. Colloid Interface Sci. 1974, 49, 16.

36. Fletcher, P. D. I.; Haswell, S. J.; Paunov, V. N. Analyst 1999, 124, 1273-1282.

37. Harmon, B. J.; Patterson, D. H.; Regnier, F. E. Anal. Chem. 1993, 65, 2655.

38. Harmon, B. J.; Leesong, I.; Regnier, F. E. Anal. Chem. 1994, 66, 3797.

39. Regnier, F. E.; Patterson, D. H.; Harmon, B. J. TrAC 1995, 14, 177.

40. Patterson, D. H.; Harmon, B. J.; Regnier, F. E. J. Chromatogr. A 1996, 732, 119.

41. Fletcher, P. D. I.; Haswell, S. J.; Zhang, X. Lab-on-a-Chip 2001, 2, 115.

42. Jacobson, S. C.; Hergenroder, R.; Koutny, L. B.; Warmack, R. J.; Ramsey, J. M. Anal. Chem. 1994, 66, 1107.

43. Ermakov, S. V.; Jacobson, S. C.; Ramsey, J. M. In Micro Total Analysis Systems '98; Harrison, D. J., Van den Berg, A., Eds.; Kluwer: Dordrecht, 1998; p 149.

44. Knight, J. B.; Vishwanath, A.; Brody, J. P.; Austin, R. H. Phys. Rev. Lett. 1998, 80, 3863.

45. Patankar, N. A.; Hu, H. H. Anal. Chem. 1998, 70, 1870.

46. Ermakov, S. V.; Jacobson, S. C.; Ramsey, J. M. Anal. Chem. 2000, 72, 3512.

47. Kamholz, A. E.; Weigl, B. H.; Finlayson, B. A.; Yager, P. Anal. Chem. 1999, 71, 5340.

48. Jacobson, S. C.; McKnight, T. E.; Ramsey, J. M. Anal. Chem. 1999, 71, 4455.

49. Polson, N. A.; Hayes, M. A. Anal. Chem. 2001, 73, 313A.

50. Kenis, P. J. A.; Ismagilov, R. F.; Whitesides, G. M. Science 1999, 285, 83.

51. James, A. D.; Robinson, B. H. J. Chem. Soc., Faraday Trans. 1 1978, 74, 10. 
52. Salimi-Moosavi, H.; Tang, T.; Harrison, D. J. J. Am. Chem. Soc. 1997, 119, 8716.

53. Doku, G. N.; Haswell, S. J.; McCreedy, T.; Greenway, G. M. Analyst 2001, 126, 14.

54. Burns, J. R.; Ramshaw, C. G. IMRET 4: Fourth International Conference of Micro Reaction Technology Topical Conference Proceedings AIChE Spring National Meeting, March 5-9, Atlanta GA, USA, 2000; p 133.

55. Skelton, V.; Greenway, G. M.; Haswell, S. J.; Styring, P.; Morgan, D. O.; Warrington, B.; Wong, S. Y. F. Analyst 2001, 126, 7 .

56. Skelton, V.; Greenway, G. M.; Haswell, S. J.; Styring, P.; Morgan, D. O.; Warrington, B.; Wong, S. Y. F. Analyst 2001, 126, 11.

57. Sands, M.; Haswell, S. J.; Kelly, S. M.; Skelton, V.; Morgan, D. O.; Styring, P.; Warrington, B. H. Lab on a Chip 2001, 1, 64.

58. Wiles, C.; Watts, P.; Haswell, S. J.; Pombo-Villar, E. Lab on a Chip 2001, 1, 100.

59. Wiles, C.; Watts, P.; Haswell, S. J.; Pombo-Villar, E. Lab on a Chip 2002 in press.

60. Lu, H.; Schmidt, M. A.; Jenson, K. F. Lab on a Chip 2001, 1, 22.

61. Pitts, J. N.; Letsinger, R. L.; Taylor, R. P.; Patterson, J. M.; Recktenwald, G.; Martin, R. B. J. Am. Chem. Soc. 1959, 81, 1068.

62. Hisamoto, H.; Saito, T.; Tokeshi, M.; Hibara, A.; Kitamori, T. J. Chem. Soc., Chem. Commun. 2001, 2662.

63. Watts, P.; Wiles, C.; Haswell, S. J.; Pombo-Villar, E.; Styring, P. J. Chem. Soc., Chem. Commun. 2001, 990.

64. Carpino, L. A.; Han, G. Y. J. Org. Chem. 1972, 37, 3404.

65. Carpino, L. A.; Cohen, B. J.; Stephens, K. E.; Sadat-Aalaee, S. Y.; Tien, J.-H.; Langridge, D. E. J. Org. Chem. 1986, 51, 3732 .
66. Chan, W. C.; Bycroft, B. W.; Evans, D. J.; White, P. D. J. Chem. Soc., Chem. Commun. 1995, 2209.

67. Greenway, G. M.; Haswell, S. J.; Morgan, D. O.; Skelton, V.; Styring, P. Sens. Actuators, B 2000, 63, 153.

68. Wilson, N. G.; McCreedy, T. J. Chem. Soc., Chem. Commun. 2000, 733.

69. Haswell, S. J.; O’Sullivan, B.; Styring, P. Lab on a Chip 2001, $1,164$.

70. Dietzsch, E.; Hönicke, D.; Fichtner, M.; Schubert, K. IMRET 4: 4th International Conference of Micro Reaction Technology Topical Conference Proceedings, AIChE Spring National Meeting, March 5-9, 2000; p 89.

71. Rozen, S. Acc. Chem. Res. 1996, 21, 307.

72. Purrington, S. T.; Kagen, B. S.; Patrick, T. B. Chem. Rev. 1986, 86, 997.

73. Chambers, R. D.; Spink, R. C. H. J. Chem. Soc., Chem. Commun. 1999, 883.

74. Chambers, R. D.; Holling, D.; Spink, R. C. H.; Sandford, G. Lab on a Chip 2001, 1, 132.

75. Chambers, R. D.; Greenhall, M. P.; Hutchinson, J. Tetrahedron 1996, 52, 1.

76. Chambers, R. D.; Grievson, B.; Drakesmith, F. G.; Powell, R. L. J. Fluorine Chem. 1985, 29, 323.

77. de Mas, N.; Jackman, R. J.; Schmidt, M. A.; Jenson, K. F. IMRET 5: Proceedings of the Fifth International Conference on Microreaction Technology, Springer: Berlin, 2002; p 60.

78. Srinivasan, R.; Hsing, I.-M.; Berger, P. E.; Jensen, K. F.; Firebaugh, S. L.; Schmidt, M. A.; Harold, M. P.; Lerou, J. J.; Ryley, J. F. AIChE J. 1997, 43 (11), 3059.

79. Hessel, V.; Ehrfeld, W.; Golbig, K.; Hofman, C.; Jungwirth, S.; Löwe, H.; Richter, T.; Storz, M.; Wolf, A. IMRET 3: Proceedings of the Third Conference on Microtechnology, 2000; p 151. 


\section{Biographical sketch}

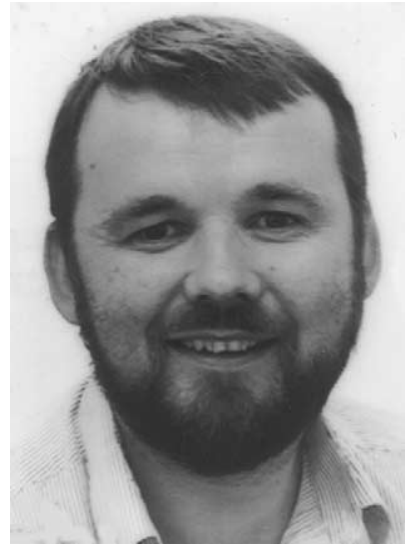

Paul Fletcher is Professor of Physical Chemistry at the University of Hull. He leads the Surfactant \& Colloid Research Group within the Department of Chemistry (see www.hull.ac.uk/scg) and his research interests include micro-reactors, microwaves and the surface and colloid chemistry of surfactant systems such as micelles, monolayers, microemulsions, emulsions and foams and reactions in complex media. He has published over 120 papers in these areas.

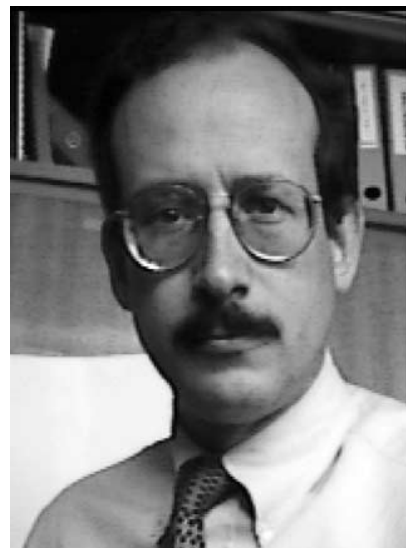

Esteban Pombo-Villar, Ph.D., C.Chem FRSC. Born in Bogotá, Colombia, May 24, 1958. Studies at the National University of Colombia, Bogotá, then at the University of Warwick, and University of Newcastle upon Tyne. M.Sc. and Ph.D. studies with Professor B. T.Golding, post-doctoral studies at ETH-Zurich with Professor A. Eschenmoser. Chaired the ECSOC-2 (1998) and ECSOC-3 (1999) electronic conferences, former Editor-inChief of Molecules. Employed with Sandoz Pharma as laboratory Head in Nervous System Research from 1988, now in Novartis Pharma AG, in Basel, Switzerland, where he is Manager of External Collaborations for Nervous System Research.

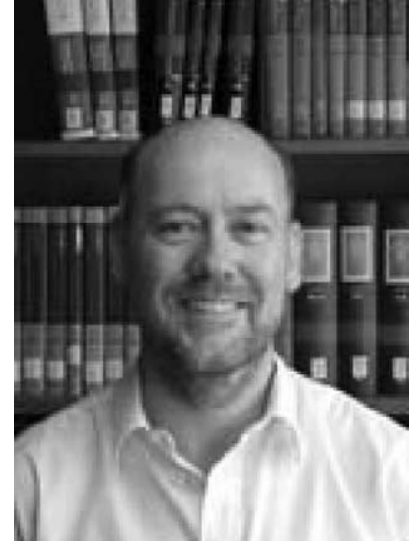

Stephen J. Haswell is Professor of Analytical Chemistry at the University of Hull. His current research activities are in the areas of micro reactors including analytical developments, microwave enhanced reaction chemistry, trace elemental speciation and process analysis. He is author of over 100 research papers, a number of books and patents and is widely known nationally and internationally for his enthusiastic lectures. For a number of years one of the underlying principles of Professor Haswell's research has been to break down the barriers that exist in science, in particular, the integration of analytical science with main line chemistry. Many of these ideals are encompassed in his research into micro chemical reactors, the subject of this article.

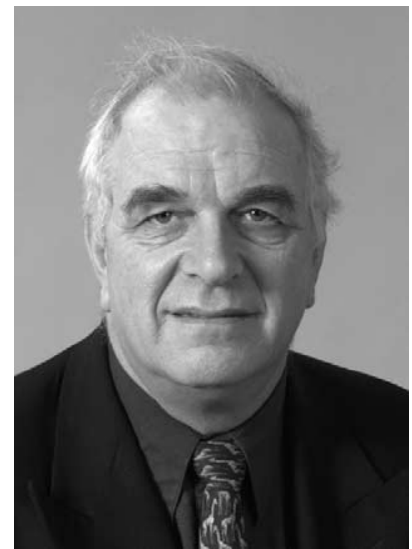

Brian H. Warrington has worked in various drug discovery roles and projects in GlaxoSmithKline Pharamcueticals and its forebears since 1965. Since 1996 his interests have concentrated on high throughput technologies for chemical synthesis and drug discovery. Currently, he is VP Technology Development, Chemistry for GSK. 


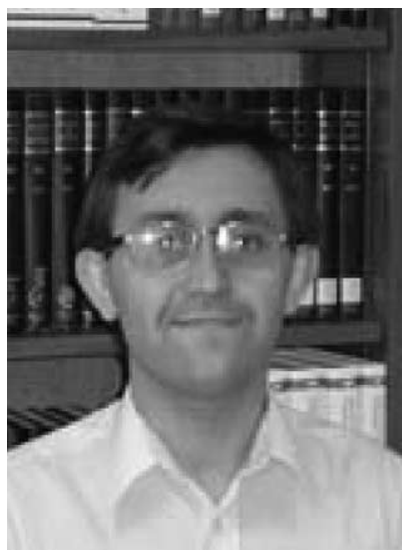

Paul Watts graduated from the University of Bristol in 1995 with a first class B.Sc. in chemistry. He continued his studies at Bristol, obtaining a Ph.D. in bio-organic chemistry under the supervision of Professor Tom Simpson FRS and Professor Chris Willis. His Ph.D. focussed on the synthesis of isotopically labelled compounds, for use in determination of biosynthetic pathways to polyketide-derived natural products. Paul subsequently worked as a postdoctoral research associate, with Professor Steve Haswell, at the University of Hull. During this period Paul investigated organic synthesis in micro reactors. In February 2002, Paul was appointed as a lecturer at the University of Hull. He is interested in organic chemistry and electrosynthesis in micro reactors.

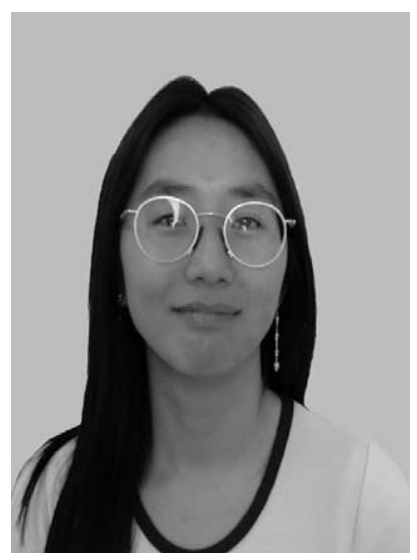

Stephanie Y. F. Wong is a Senior Scientist at GlaxoSmithKline Pharmaceuticals in the Technology Development Department. She has worked in the pharmaceutical industry for over 10 years in several therapeutic areas and on targets such as nitric oxide synthase and serotonin receptors. Since the start of 1998 she has been working in the microfluidics area (Microsystems Technology) on novel methods to enhancing the drug discovery and optimisation process.

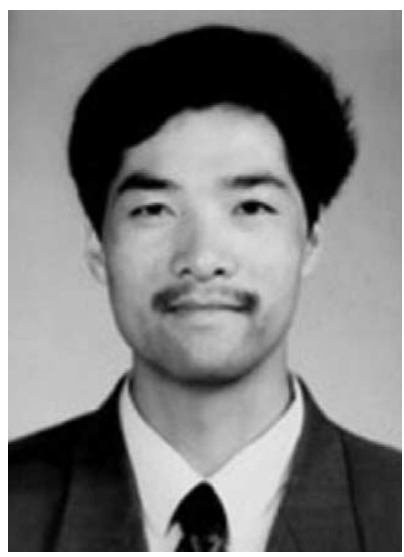

Xunli Zhang graduated from Northwest University in Xi' an, China, with a B.Sc. and an M. Eng. in Chemical Engineering. He obtained his Ph.D., studying on heterogeneous catalysis using microwave and conventional heating, from Imperial College of Science, Technology and Medicine of the University of London in 2000. Dr Xunli Zhang is currently working at the University of Hull in the Microreactor Research Group, aiming to model the behaviour of fluidics and chemical reactions in microreactors, develop methods of monitoring and control of microreactors, and integrate automated micro chemical systems. 